\title{
Weighted spanning trees on some self-similar graphs
}

\author{
Daniele D'Angeli \\ Departamento de Matemáticas \\ Universidad de los Andes \\ Carrera 1, 18A - 70 Bogotá, Colombia \\ dangeli@uniandes.edu.co
}

\author{
Alfredo Donno \\ Dipartimento di Matematica \\ Sapienza Università di Roma \\ Piazzale A. Moro, 200185 Roma, Italia \\ donno@mat.uniroma1.it
}

Submitted: Aug 11, 2010; Accepted: Dec 20, 2010; Published: Jan 12, 2011

Mathematics Subject Classification: 05A15, 05C22, 20E08, 05C25

\begin{abstract}
We compute the complexity of two infinite families of finite graphs: the Sierpiński graphs, which are finite approximations of the well-known Sierpiński gasket, and the Schreier graphs of the Hanoi Towers group $H^{(3)}$ acting on the rooted ternary tree. For both of them, we study the weighted generating functions of the spanning trees, associated with several natural labellings of the edge sets.
\end{abstract}

\section{Introduction}

The enumeration of spanning trees in a finite graph is largely studied in the literature, and it has many applications in several areas of Mathematics as Algebra, Combinatorics, Probability and of Theoretical Computer Science.

Given a connected finite graph $Y=(V(Y), E(Y))$, where $V(Y)$ and $E(Y)$ denote the vertex set and the edge set of $Y$, respectively, a spanning tree of $Y$ is a subgraph of $Y$ which is a tree and whose vertex set coincides with $V(Y)$.

The number of spanning trees of a graph $Y$ is called the complexity of $Y$ and is denoted by $\tau(Y)$. The famous Kirchhoff's Matrix-Tree Theorem (1847) states that $\tau(Y)$ is equal to (the constant value of) any cofactor of the Laplace matrix of $Y$, which is obtained as the difference between the degree matrix of $Y$ and its adjacency matrix. Equivalently, $\tau(Y) \cdot|V(Y)|$ is given by the product of all nonzero eigenvalues of the Laplace matrix of $Y$.

It is interesting to study complexity when the system grows. More precisely, given a sequence $\left\{Y_{n}\right\}_{n \geq 1}$ of finite graphs with complexity $\tau\left(Y_{n}\right)$, such that $\left|V\left(Y_{n}\right)\right| \rightarrow \infty$, the limit

$$
\lim _{\left|V\left(Y_{n}\right)\right| \rightarrow \infty} \frac{\log \tau\left(Y_{n}\right)}{\left|V\left(Y_{n}\right)\right|},
$$


when it exists, is called the asymptotic growth constant of the spanning trees of $\left\{Y_{n}\right\}_{n \geq 1}$ (see [13]).

A spanning $k$-forest of $Y$ is a subgraph of $Y$ which is a $k$-forest, i.e., it is a forest with $k$ connected components, and its vertex set coincides with $V(Y)$.

The enumeration of spanning subgraphs, in general, for a graph $Y$, is also strictly related to the Tutte polynomial $T_{Y}(x, y)$ of the graph: more precisely, it is known that $T_{Y}(1,1)$ equals the complexity of $Y, T_{Y}(2,1)$ equals the number of spanning forests of $Y$, and $T_{Y}(1,2)$ is the number of its connected spanning subgraphs (see [5,9], where this analysis is developed for the finite Sierpiński graphs and for other examples of finite graphs associated with the action of automorphisms groups of rooted regular trees).

A finer invariant of the graph $Y$ is a finite abelian group $\Phi(Y)$, whose order is exactly the complexity of $Y$. This group occurs in the literature under different names, depending on the context. It was introduced in [1] as the Picard group of $Y$ (or the Jacobian of $Y$ ), whereas it is shown in [4] that the Picard group is isomorphic to the group of critical configurations of the chip-firing game on $Y$. As any finite abelian group, $\Phi(Y)$ can be decomposed into direct sum of invariant factors. The dependence of this decomposition on the properties of $Y$ has been studied by several authors, (see, e.g., [12]), but not much is known so far. Explicit computations have been performed for certain families of graphs.

In many optimization problems it is often useful to find a minimal spanning tree of a weighted graph. Hence, it is interesting to study spanning trees when a weight function on $E(Y)$ is defined. In order to do this, we introduce the formal variables $w_{e}$, with $e \in E(Y)$. These variables will be regarded as weights on the edges of the graph, so that we can assume that they take only positive real values. Put $w=\left\{w_{e}\right\}_{e \in E(Y)}$ and let $\mathcal{T}$ be the set of all spanning trees of $Y$. With each spanning tree $t \in \mathcal{T}$, we can associate the weight function

$$
W(t):=\prod_{e \in E(t)} w_{e}
$$

i.e., the product of the formal variables $w_{e}$ associated with the edges of $Y$ belonging to $E(t)$. Then, the weighted generating function of the spanning trees of $Y$ is the polynomial on the formal variables $\left\{w_{e}\right\}_{e \in E(Y)}$, given by

$$
T(w):=\sum_{t \in \mathcal{T}} W(t) .
$$

It follows from the definition that, if $w_{e}=1$ for each $e \in E(Y)$, the generating function yields the complexity of the graph, since in this case one has $W(t)=1$, for each $t \in \mathcal{T}$.

In this paper, we will study weighted spanning trees on two infinite families of finite graphs very close to each other: the Sierpiński graphs, which are finite approximations of the famous Sierpiński gasket, and the Schreier graphs of the Hanoi Towers group $H^{(3)}$, which is an example of a self-similar group (see Definition 1.2 below).

We recall some basic facts about self-similar groups. Let $T_{q}$ be the infinite regular rooted tree of degree $q$, i.e., the rooted tree in which each vertex has $q$ children. Each vertex of the $n$-th level of the tree can be regarded as a word of length $n$ in the alphabet $X=\{0,1, \ldots, q-1\}$. Now let $G<\operatorname{Aut}\left(T_{q}\right)$ be a group acting on $T_{q}$ by automorphisms 
generated by a finite symmetric set of generators $S$. Suppose, moreover, that the action is transitive on each level of the tree.

Definition 1.1. The $n$-th Schreier graph $\Sigma_{n}$ of the action of $G$ on $T_{q}$, with respect to the generating set $S$, is a graph whose vertex set coincides with the set of vertices of the $n$-th level of the tree, and two vertices $u, v$ are adjacent if and only if there exists $s \in S$ such that $s(u)=v$. If this is the case, the edge joining $u$ and $v$ is labelled by $s$.

The vertices of $\Sigma_{n}$ are labelled by words of length $n$ in $X$ and the edges are labelled by elements of $S$. The Schreier graph is thus a regular graph of degree $|S|$ with $q^{n}$ vertices, and it is connected since the action of $G$ is level-transitive.

Definition $1.2([14])$. A finitely generated group $G<A u t\left(T_{q}\right)$ is self-similar if, for all $g \in G, x \in X$, there exist $h \in G, y \in X$ such that

$$
g(x w)=y h(w)
$$

for all finite words $w$ in the alphabet $X$.

Self-similarity implies that $G$ can be embedded into the wreath product $\operatorname{Sym}(q)$ ₹ $G=$ $\operatorname{Sym}(q) \ltimes G^{q}$, where $\operatorname{Sym}(q)$ denotes the symmetric group on $q$ elements, so that any automorphism $g \in G$ can be represented as

$$
g=\alpha\left(g_{0}, \ldots, g_{q-1}\right),
$$

where $\alpha \in \operatorname{Sym}(q)$ describes the action of $g$ on the first level of $T_{q}$ and $g_{i} \in G, i=$ $0, \ldots, q-1$, is the restriction of $g$ on the full subtree of $T_{q}$ rooted at the vertex $i$ of the first level of $T_{q}$ (observe that any such subtree is isomorphic to $T_{q}$ ). Hence, if $x \in X$ and $w$ is a finite word in $X$, we have

$$
g(x w)=\alpha(x) g_{x}(w) .
$$

The class of self-similar groups contains many interesting examples of groups which have exotic properties: among them, we mention the first Grigorchuk group, which yields the simplest solution of the Burnside problem (an infinite, finitely generated torsion group) and the first example of a group of intermediate growth (see [10] for a detailed account and further references). In the last decades, automorphisms groups of rooted trees have been largely investigated: R. Grigorchuk and a number of coauthors have developed a new exciting direction of research focusing on finitely generated groups acting by automorphisms on rooted trees [3]. They proved that these groups have deep connections with the theory of profinite groups and with complex dynamics. In particular, for many examples of groups belonging to this class, the property of self-similarity is reflected on fractalness of some limit objects associated with them [14].

Since the Schreier graphs are determined by group actions, their edges are naturally labelled by the generators of the acting group and it takes sense to study weighted spanning trees on them, with respect to this labelling.

The paper is structured as follows. In Section 2, we study weighted spanning trees on finite approximations of the well-known Sierpiński gasket, endowed with three different edge labellings: 
- the "rotational-invariant" labelling, whose special symmetry allows to explicitly compute the generating function of the spanning trees (Theorem 2.2) and to perform a statistical analysis about the number of edges, with a fixed label, occurring in a random spanning tree of the graph (Proposition 4.1);

- the "directional" labelling, where the weights depend on the direction of the edges; for this model, the weighted generating function of the spanning trees is described via the iteration of a polynomial map (Theorem 2.8);

- the "Schreier" labelling, strictly related to the labelling of the Schreier graphs of the Hanoi Towers group $H^{(3)}$; also in this case, the weighted generating function of the spanning trees is described via the iteration of a polynomial map (Theorem 2.12).

In all these models we follow a combinatorial approach. The self-similar structure of the graph (in the sense of [16]) allows to study both unweighted and weighted subgraphs recursively. More precisely, we introduce three different generating functions associated with spanning trees, 2-spanning forests, 3-spanning forests and, using self-similarity, we are able to establish recursive relations (Theorems 2.1, 2.6 and 2.10) and to give an explicit description of them (Theorems 2.2, 2.8 and 2.12). More generally, the self-similar structure of a graph turns out to be a powerful tool for investigating many combinatorial and statistical models on it: see, for instance, $[7,8,15,16,17]$.

In Section 3, we consider the Schreier graphs of the Hanoi Towers group $H^{(3)}$, whose action on the ternary tree models the famous Hanoi Towers game on three pegs (see [11]), endowed with the natural edge labelling coming from the action of its generators. Even if these graphs also have a self-similar structure, the combinatorial approach used in the case of the Sierpiński graphs seems to be much harder here. Therefore, our technique consists in using a weighted version of the Kirchhoff's Theorem: we construct the Laplace matrix by using the self-similar presentation of the generators of the group, which is impossible in the case of Sierpiński graphs, where there is no group structure. In this case, the generating function is described in terms of iterations of a rational map (Theorem 3.5): this kind of approach already appears in $[2,11]$ (see also [8], where we use the same strategy to compute the partition function of the dimer model on the Schreier graphs of the Hanoi Towers group).

\section{Spanning trees on the Sierpiński graphs}

The problem of enumeration of spanning trees in Sierpiński graphs was largely treated in literature (see, for instance, $[6,15]$ ). We consider here three different labellings of the edges of these graphs and write down the associated generating function of the spanning trees. In all the models, the self-similarity of the graphs plays a crucial role to study the problem recursively. The description of the generating function strongly depends on the symmetry of the labelling of the graph: as we will see, in the first model that we consider, which is invariant under rotation, we are able to give an explicit formula for it; in the two 
remaining models, where we do not have invariance under the action of any symmetry group, the generating function is described via the iteration of two polynomial maps.

\subsection{First model: "Rotational-invariant"labelling}

Let $\Gamma_{1}$ be the graph in the following picture.

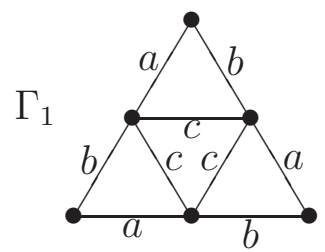

For each $n \geq 1$, we define, by recurrence, the graph $\Gamma_{n+1}$ as the graph obtained by partitioning an equilateral triangle in four smaller equilateral triangles and by putting in each corner a copy of $\Gamma_{n}$. Observe that this labelling of the graph is invariant with respect to the rotation of $\frac{2 \pi}{3}$. We represent in the following picture the graph $\Gamma_{2}$.

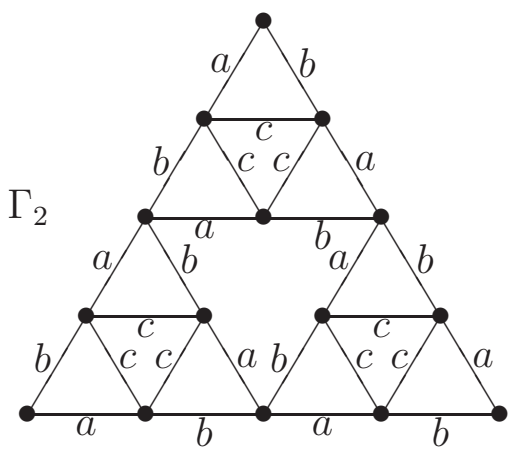

We want to study weighted spanning trees on the graphs $\left\{\Gamma_{n}\right\}_{n \geq 1}$. For each $n \geq 1$, we put:

- $T_{n}(a, b, c)=$ weighted generating function of the spanning trees of $\Gamma_{n}$;

- $S_{n}(a, b, c)=$ weighted generating function of the spanning 2-forests of $\Gamma_{n}$, where two fixed outmost vertices belong to the same connected component and the third outmost vertex belongs to the second connected component;

- $Q_{n}(a, b, c)=$ weighted generating function of the spanning 3 -forests of $\Gamma_{n}$, where the three outmost vertices belong to three different connected components.

Observe that, because of the rotational invariance of the labelling of the graph, the function $S_{n}(a, b, c)$ does not depend on the choice of the two outmost vertices. In what follows, we will often omit the argument $(a, b, c)$ of the weighted generating functions. 
Theorem 2.1. For each $n \geq 1$, the weighted generating functions $T_{n}(a, b, c), S_{n}(a, b, c)$ and $Q_{n}(a, b, c)$ satisfy the following equations:

$$
\begin{gathered}
T_{n+1}=6 T_{n}^{2} S_{n} \\
S_{n+1}=7 T_{n} S_{n}^{2}+T_{n}^{2} Q_{n} \\
Q_{n+1}=12 T_{n} S_{n} Q_{n}+14 S_{n}^{3},
\end{gathered}
$$

with initial conditions

$$
\begin{gathered}
T_{1}(a, b, c)=3(a+b)(a b+a c+b c)^{2} \\
S_{1}(a, b, c)=(a+b)(a+b+3 c)(a b+a c+b c) \quad Q_{1}(a, b, c)=(a+b)(a+b+3 c)^{2} .
\end{gathered}
$$

Proof. The graph $\Gamma_{n+1}$ can be represented as a triangle containing three copies of $\Gamma_{n}$.

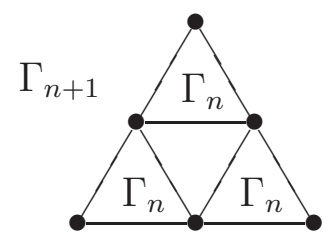

We will use the pictures
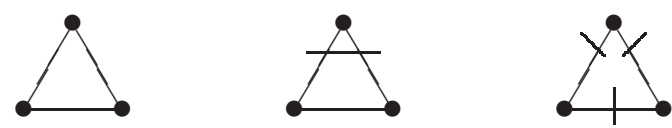

to denote, respectively, the case where in a copy of $\Gamma_{n}$ :

- the three outmost vertices are in the same connected component;

- two outmost vertices are in the same connected component and the third one is in a different connected component;

- the outmost vertices are in three different connected components.

The only way to construct a spanning tree of $\Gamma_{n+1}$ is to choose a spanning tree in two copies of $\Gamma_{n}$ and a spanning 2-forest in the third one, as in the following picture.

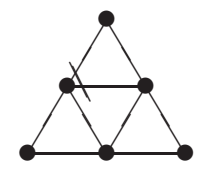

This argument proves Equation (1), where the factor 6 is given by symmetry (we have to take into account both reflections and rotations).

Next, we are going to prove Equation (2) (we analyze, for instance, the case where the leftmost and the rightmost vertices are in the same connected component). Consider the two following pictures. 

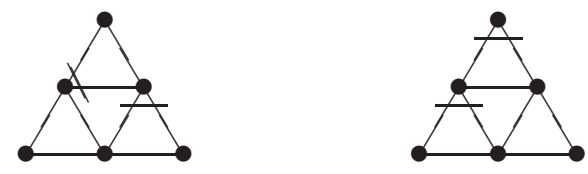

These possibilities, together with their symmetric, obtained by reflecting with respect to the vertical axis, give a contribution to $S_{n+1}$ equal to $4 T_{n} S_{n}^{2}$. Consider now the two following configurations.
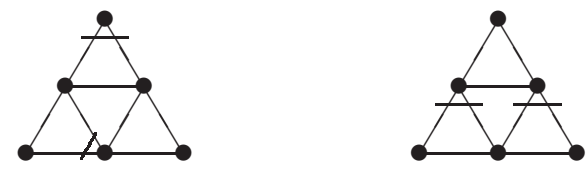

Since the picture on the left has to be considered together with its symmetric, we get a contribution to $S_{n+1}$ equal to $3 T_{n} S_{n}^{2}$. Finally, the contribution $T_{n}^{2} Q_{n}$ is described by the following picture.

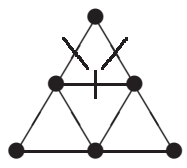

This proves Equation (2).

We have now to prove Equation (3) about $Q_{n+1}$. Consider the following situations.
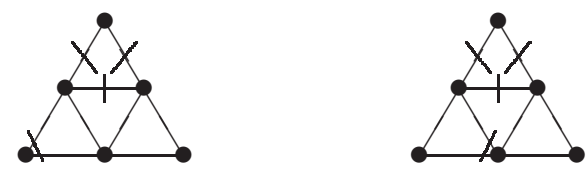

They provide, by symmetry, a contribution equal to $12 T_{n} S_{n} Q_{n}$. The following pictures give, by symmetry, a contribution of $12 S_{n}^{3}$ to $Q_{n+1}$.
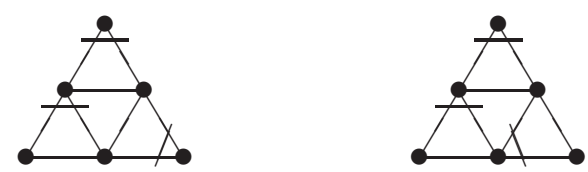

Finally, the following two pictures give a contribution of $2 S_{n}^{3}$ to $Q_{n+1}$.
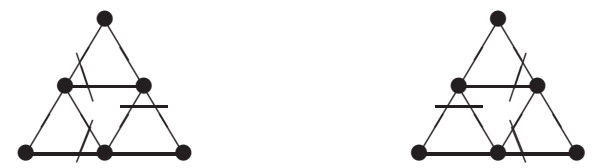

This completes the proof.

Theorem 2.2. For each $n \geq 1$, the weighted generating functions $T_{n}(a, b, c), S_{n}(a, b, c)$ and $Q_{n}(a, b, c)$ satisfying Equations (1), (2) and (3), with the initial conditions given in Theorem 2.1, are:

$$
T_{n}(a, b, c)=2^{\frac{3^{n-1}-1}{2}} 3^{\frac{3^{n}+2 n-1}{4}} 5^{\frac{3^{n-1}-2 n+1}{4}}(a+b)^{3^{n-1}}(a+b+3 c)^{\frac{3^{n-1}-1}{2}}(a b+a c+b c)^{\frac{3^{n}+1}{2}} ;
$$




$$
\begin{aligned}
& S_{n}(a, b, c)=2^{\frac{3^{n-1}-1}{2}} 3^{\frac{3^{n}-2 n-1}{4}} 5^{\frac{3^{n-1}+2 n-3}{4}}(a+b)^{3^{n-1}}(a+b+3 c)^{\frac{3^{n-1}+1}{2}}(a b+a c+b c)^{\frac{3^{n}-1}{2}} \\
& Q_{n}(a, b, c)=2^{\frac{3^{n-1}-1}{2}} 3^{\frac{3^{n}-6 n+3}{4}} 5^{\frac{3^{n-1}+6 n-7}{4}}(a+b)^{3^{n-1}}(a+b+3 c)^{\frac{3^{n-1}+3}{2}}(a b+a c+b c)^{\frac{3^{n}-3}{2}} .
\end{aligned}
$$

Proof. The proof is by induction on $n$. It is easy to verify that, for $n=1$, one gets the initial conditions given in Theorem 2.1. Then, one can check that the functions given in the claim satisfy Equations (1), (2) and (3). We omit here the explicit computations.

It follows that $T_{n}(1,1,1)=\tau\left(\Gamma_{n}\right)$; similarly, $s\left(\Gamma_{n}\right):=S_{n}(1,1,1)$ is the number of spanning 2-forests of $\Gamma_{n}$, where two fixed outmost vertices belong to the same connected component and the third outmost vertex belongs to the second connected component; $q\left(\Gamma_{n}\right):=Q_{n}(1,1,1)$ is the number of spanning 3 -forests of $\Gamma_{n}$, where the three outmost vertices belong to three different connected components.

Corollary 2.3. For each $n \geq 1$, one has:

$$
\begin{aligned}
& \text { 1. } \tau\left(\Gamma_{n}\right)=2^{\frac{3^{n}-1}{2}} 3^{\frac{3^{n+1}+2 n+1}{4}} 5^{\frac{3^{n}-2 n-1}{4}} ; \\
& \text { 2. } s\left(\Gamma_{n}\right)=2^{\frac{3^{n}-1}{2}} 3^{\frac{3^{n+1}-2 n-3}{4}} 5^{\frac{3^{n}+2 n-1}{4}} ; \\
& \text { 3. } q\left(\Gamma_{n}\right)=2^{\frac{3^{n}-1}{2}} 3^{\frac{3^{n+1}-6 n-3}{4}} 5^{\frac{3^{n}+6 n-1}{4}} \text {. }
\end{aligned}
$$

In particular, the asymptotic growth constant of the spanning trees of $\Gamma_{n}$ is $\frac{1}{3} \log 2+$ $\frac{1}{2} \log 3+\frac{1}{6} \log 5$.

Proof. It suffices to evaluate the weighted generating functions described in Theorem 2.2 for $a=b=c=1$. The asymptotic growth constant is then obtained as the limit

$$
\lim _{n \rightarrow \infty} \frac{\log \left(\tau\left(\Gamma_{n}\right)\right)}{\left|V\left(\Gamma_{n}\right)\right|},
$$

where $\left|V\left(\Gamma_{n}\right)\right|=\frac{3}{2}\left(3^{n}+1\right)$ is the number of vertices of $\Gamma_{n}$, for each $n \geq 1$.

Remark 2.4. The same values of the complexity and of the asymptotic growth constant have been found in [6] and [15], where the authors study unweighted spanning trees of $\Gamma_{n}$.

\subsection{Second model: "directional" labelling}

Consider now a new sequence of graphs $\left\{\Gamma_{n}\right\}_{n \geq 1}$, which coincide, as unweighted graphs, with the graphs studied in Section 2.1, and whose edges are endowed with a new labelling, that we call directional labelling. It is clear that an edge of $\Gamma_{n}$ can point in three different directions: up (from left to right), down (from left to right) or horizontal. Then, we label by $a$ each edge pointing up, by $b$ each horizontal edge, and by $c$ each edge pointing down, where, as usual, $a, b, c \in \mathbb{R}_{+}$. Here we draw the three first examples. 


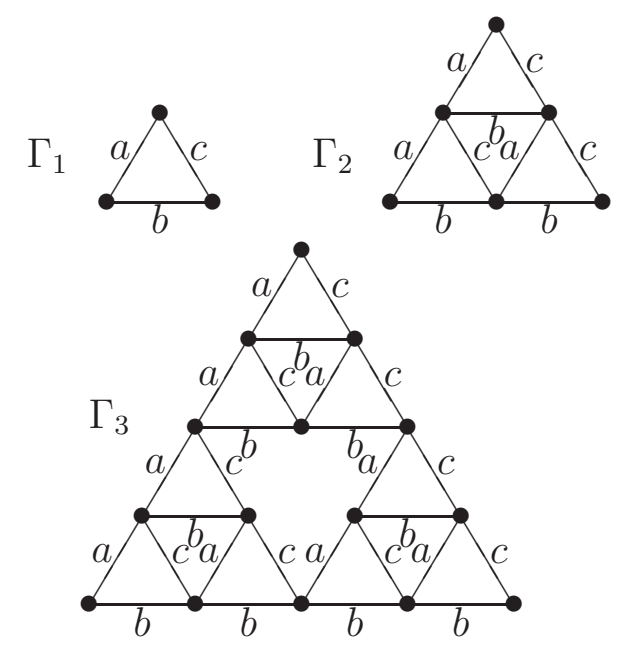

Remark 2.5. Observe that the indices are now shifted by 1 with respect to the case of the rotational-invariant labelling considered in Section 2.1: the reason is that a rotationalinvariant labelling using three labels $a, b$ and $c$ cannot be defined on a simple triangle.

In this section, we study the weighted spanning trees of the graph $\Gamma_{n}$ endowed with the directional labelling. For each $n \geq 1$, we put:

- $T_{n}(a, b, c)=$ weighted generating function of the spanning trees of $\Gamma_{n}$;

- $U_{n}(a, b, c)=$ weighted generating function of the spanning 2-forests of $\Gamma_{n}$, where the leftmost and the rightmost vertices belong to the same connected component, and the upmost vertex belongs to the second connected component. Similarly, by rotation, we define $R_{n}(a, b, c)$ (respectively $L_{n}(a, b, c)$ ) for the spanning 2 -forests of $\Gamma_{n}$, where the rightmost (respectively leftmost) vertex is not in the same connected component containing the two other outmost vertices;

- $Q_{n}(a, b, c)=$ weighted generating function of the spanning 3-forests of $\Gamma_{n}$, where the three outmost vertices belong to three different connected components.

Observe that, in this model, we need to introduce three different functions $U_{n}(a, b, c)$, $R_{n}(a, b, c)$ and $L_{n}(a, b, c)$, since the edge labelling is not invariant with respect to a rotation of $\frac{2 \pi}{3}$ as in the previous case. On the other hand it is clear that, for each $n \geq 1$, one has $U_{n}(1,1,1)=R_{n}(1,1,1)=L_{n}(1,1,1)$ and this common value is equal to $S_{n-1}(1,1,1)$, where $S_{n}(a, b, c)$ is the generating function introduced in Section 2.1. In what follows, we will often omit the argument $(a, b, c)$ of the generating functions.

Theorem 2.6. For each $n \geq 1$, the weighted generating functions $T_{n}(a, b, c), U_{n}(a, b, c)$, $R_{n}(a, b, c), L_{n}(a, b, c)$ and $Q_{n}(a, b, c)$ satisfy the following equations:

$$
\begin{gathered}
T_{n+1}=2 T_{n}^{2}\left(U_{n}+R_{n}+L_{n}\right) \\
U_{n+1}=T_{n} U_{n}\left(2 R_{n}+2 L_{n}+3 U_{n}\right)+T_{n}^{2} Q_{n}
\end{gathered}
$$




$$
\begin{aligned}
& R_{n+1}=T_{n} R_{n}\left(2 L_{n}+2 U_{n}+3 R_{n}\right)+T_{n}^{2} Q_{n} \\
& L_{n+1}=T_{n} L_{n}\left(2 R_{n}+2 U_{n}+3 L_{n}\right)+T_{n}^{2} Q_{n} \\
Q_{n+1} & =4 T_{n} Q_{n}\left(U_{n}+R_{n}+L_{n}\right) \\
& +2\left(U_{n}^{2}\left(R_{n}+L_{n}\right)+R_{n}^{2}\left(L_{n}+U_{n}\right)+L_{n}^{2}\left(R_{n}+U_{n}\right)\right) \\
& +2 U_{n} R_{n} L_{n},
\end{aligned}
$$

with initial conditions

$$
\begin{gathered}
T_{1}(a, b, c)=a b+a c+b c \quad U_{1}(a, b, c)=b \quad R_{1}(a, b, c)=a \\
L_{1}(a, b, c)=c \quad Q_{1}(a, b, c)=1 .
\end{gathered}
$$

Proof. It is easy to check that the initial conditions hold. Then, the proof of each recursive equation follows the same strategy as in Theorem 2.1.

Remark 2.7. Observe that, by replacing $U_{n}, R_{n}$ and $L_{n}$ with $S_{n}$, one finds again the equations given for the rotational-invariant model in Theorem 2.1.

In order to get explicit solutions of the equations given in Theorem 2.6, we put

$$
\begin{gathered}
\phi_{1}(a, b, c)=a b+a c+b c \quad \phi_{2}(a, b, c)=a+b+c \\
f(a, b, c)=3 a^{2} b+3 a b^{2}+3 a^{2} c+3 a c^{2}+3 b^{2} c+3 b c^{2}+7 a b c
\end{gathered}
$$

and let us define the function $F: \mathbb{R}^{3} \longrightarrow \mathbb{R}^{3}$ as

$$
F(x, y, z)=\left(F_{1}(x, y, z), F_{2}(x, y, z), F_{3}(x, y, z)\right)
$$

with

$$
\begin{gathered}
F_{1}(x, y, z)=3 x^{2}+3 x z+3 x y+y z \quad F_{2}(x, y, z)=3 y^{2}+3 x y+3 y z+x z \\
F_{3}(x, y, z)=3 z^{2}+3 x z+3 y z+x y .
\end{gathered}
$$

Moreover, we denote by $F_{i}^{(k)}(a, b, c)$ the $i$-th coordinate of the vector

$$
F^{(k)}=F(\ldots F(F(a, b, c))),
$$

where the function $F$ is iterated $k$ times. Note that $F_{i}^{(1)}(a, b, c)=F_{i}(a, b, c)$, for each $i=1,2,3$.

Finally, for each $k \geq 3$, put $\phi_{k}(a, b, c)=\phi_{k-1}\left(F_{1}(a, b, c), F_{2}(a, b, c), F_{3}(a, b, c)\right)$, so that

$$
\phi_{k}(a, b, c)=\phi_{2}\left(F^{(k-2)}(a, b, c)\right) \text {. }
$$


Theorem 2.8. The weighted generating functions $T_{n}(a, b, c), U_{n}(a, b, c), R_{n}(a, b, c)$, $L_{n}(a, b, c)$ and $Q_{n}(a, b, c)$ satisfying Equations (4), (5), (6), (7) and (8), with the initial conditions given in Theorem 2.6, are:

$$
\begin{aligned}
& T_{n}(a, b, c)=2^{\frac{3^{n}+6 n-9}{12}} \prod_{k=1}^{n} \phi_{k}^{\frac{3^{n-k+1}+3}{6}}(a, b, c), \quad \text { for each } n \geq 1 ; \\
& U_{n}(a, b, c)=2^{\frac{3^{n}-6 n+3}{12}} \prod_{k=1}^{n-1} \phi_{k}^{{\frac{3^{n-k+1}-3}{6}}^{6}}(a, b, c) F_{2}^{(n-1)}(a, b, c), \quad \text { for each } n \geq 2 \\
& R_{n}(a, b, c)=2^{\frac{3^{n}-6 n+3}{12}} \prod_{k=1}^{n-1} \phi_{k}^{\frac{3}{n-k+1}-3^{6}}(a, b, c) F_{1}^{(n-1)}(a, b, c), \quad \text { for each } n \geq 2 \\
& L_{n}(a, b, c)=2^{\frac{3^{n}-6 n+3}{12}} \prod_{k=1}^{n-1} \phi_{k}^{\frac{3}{n-k+1}-3^{6}}(a, b, c) F_{3}^{(n-1)}(a, b, c), \quad \text { for each } n \geq 2 \\
& Q_{n}(a, b, c)=2^{\frac{3^{n}-18 n+39}{12}} \prod_{k=1}^{n-2} \phi_{k}^{\frac{3^{n-k+1}-9}{6}}(a, b, c) f\left(F^{(n-2)}(a, b, c)\right) \quad \text { for each } n \geq 3,
\end{aligned}
$$

with $U_{1}(a, b, c)=b, R_{1}(a, b, c)=a, L_{1}(a, b, c)=c, Q_{1}(a, b, c)=1$ and $Q_{2}(a, b, c)=$ $2 f(a, b, c)$.

Proof. The proof works by induction on $n$. One can directly find:

$$
\begin{gathered}
T_{1}(a, b, c)=\phi_{1}(a, b, c) \quad U_{2}(a, b, c)=\phi_{1}(a, b, c) F_{2}(a, b, c) \\
R_{2}(a, b, c)=\phi_{1}(a, b, c) F_{1}(a, b, c) \quad L_{2}(a, b, c)=\phi_{1}(a, b, c) F_{3}(a, b, c) \\
Q_{3}(a, b, c)=2 \phi_{1}^{3}(a, b, c) f\left(F_{1}(a, b, c), F_{2}(a, b, c), F_{3}(a, b, c)\right),
\end{gathered}
$$

and so the basis of induction holds. We only prove the assertion for $T_{n}(a, b, c)$, by showing that Equation (4) is satisfied (the computations in the other cases are similar but more complicated). One has:

$$
\begin{aligned}
2 T_{n}^{2}\left(U_{n}+R_{n}+L_{n}\right)= & 2 \cdot 2^{\frac{3^{n}+6 n-9}{6}} \prod_{k=1}^{n} \phi_{k}^{\frac{3^{n-k+1}+3}{3}}(a, b, c) \cdot 2^{\frac{3^{n}-6 n+3}{12}} \prod_{k=1}^{n-1} \phi_{k}^{\frac{3^{n-k+1}-3}{6}}(a, b, c) \\
& \left(F_{2}^{(n-1)}(a, b, c)+F_{1}^{(n-1)}(a, b, c)+F_{3}^{(n-1)}(a, b, c)\right) \\
= & 2^{\frac{3^{n+1}+6 n-3}{12}} \prod_{k=1}^{n} \phi_{k}^{\frac{3^{n-k+2}+3}{6}}(a, b, c) \\
& \cdot\left(F_{2}^{(n-1)}(a, b, c)+F_{1}^{(n-1)}(a, b, c)+F_{3}^{(n-1)}(a, b, c)\right) \\
= & 2^{\frac{3^{n+1}+6 n-3}{12}} \prod_{k=1}^{n} \phi_{k}^{\frac{3^{n-k+2}+3}{6}}(a, b, c) \phi_{2}\left(F^{(n-1)}(a, b, c)\right) \\
= & 2^{\frac{3^{n+1}+6 n-3}{12}} \prod_{k=1}^{n+1} \phi_{k}^{\frac{3^{n-k+2}+3}{6}}(a, b, c)=T_{n+1} .
\end{aligned}
$$




\subsection{Third model: the "Schreier"labelling}

Consider the graph $\Gamma_{1}$ in the picture below and define by recurrence, for each $n \geq 1$, the graph $\Gamma_{n+1}$ as constituted by the union of three copies of $\Gamma_{n}$ in the following way: for each one of the outmost vertices of $\Gamma_{n+1}$, the corresponding copy is given by the graph $\Gamma_{n}$, reflected with respect to the bisectrix of the corresponding angle.

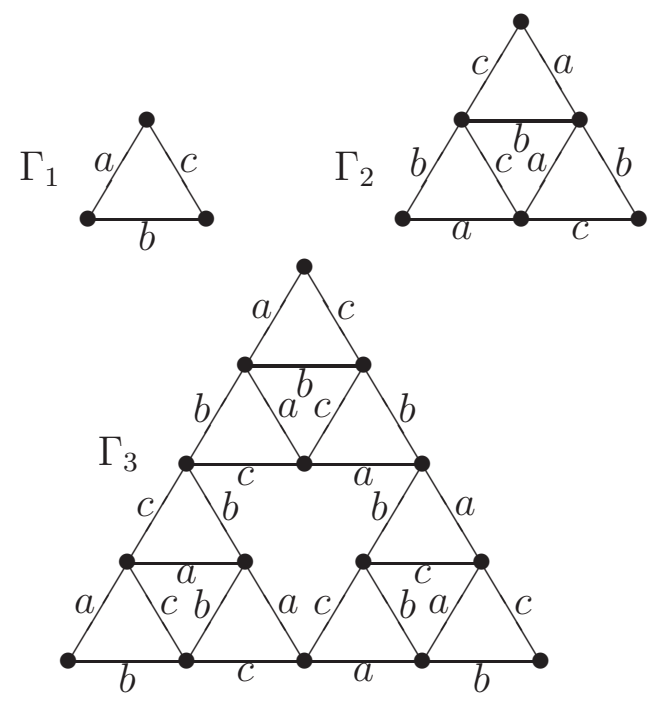

Remark 2.9. For each $n \geq 1$, the graph $\Gamma_{n}$ coincides with the graph obtained from the Schreier graph $\Sigma_{n}$ of the Hanoi Towers Group $H^{(3)}$ by deleting the three loops and by contracting all the edges joining two different elementary triangles, keeping the labels in $\Sigma_{n}$ on the remaining edges. (See Section 3.1.)

Define the generating functions $T_{n}(a, b, c), U_{n}(a, b, c), R_{n}(a, b, c), L_{n}(a, b, c)$ and $Q_{n}(a, b, c)$ to have the same meaning as in the case of the directional labelling (Section 2.2). In what follows, we will often omit the argument $(a, b, c)$ of the generating functions.

Theorem 2.10. For each $n \geq 1$, the weighted generating functions $T_{n}(a, b, c), U_{n}(a, b, c)$, $R_{n}(a, b, c), L_{n}(a, b, c)$ and $Q_{n}(a, b, c)$ satisfy the following equations:

$$
\begin{gathered}
T_{n+1}=2 T_{n}^{2}\left(U_{n}+R_{n}+L_{n}\right) \\
U_{n+1}=T_{n}\left(3 L_{n} R_{n}+U_{n} R_{n}+U_{n} L_{n}+2 U_{n}^{2}\right)+T_{n}^{2} Q_{n} \\
R_{n+1}=T_{n}\left(3 U_{n} L_{n}+U_{n} R_{n}+R_{n} L_{n}+2 R_{n}^{2}\right)+T_{n}^{2} Q_{n} \\
L_{n+1}=T_{n}\left(3 U_{n} R_{n}+L_{n} U_{n}+R_{n} L_{n}+2 L_{n}^{2}\right)+T_{n}^{2} Q_{n}
\end{gathered}
$$




$$
\begin{aligned}
Q_{n+1} & =4 T_{n} Q_{n}\left(U_{n}+R_{n}+L_{n}\right) \\
& +2\left(U_{n}^{2}\left(L_{n}+R_{n}\right)+R_{n}^{2}\left(U_{n}+L_{n}\right)+L_{n}^{2}\left(R_{n}+U_{n}\right)\right) \\
& +2 U_{n} R_{n} L_{n}
\end{aligned}
$$

with initial conditions

$$
\begin{gathered}
T_{1}(a, b, c)=a b+a c+b c \quad U_{1}(a, b, c)=b \quad R_{1}(a, b, c)=a \\
L_{1}(a, b, c)=c \quad Q_{1}(a, b, c)=1 .
\end{gathered}
$$

Proof. It is easy to check that the initial conditions hold. Then, the proof of each recursive equation follows the same strategy as in Theorem 2.1.

Remark 2.11. Observe that, by replacing $U_{n}, R_{n}$ and $L_{n}$ with $S_{n}$, one finds again the equations obtained for the rotational-invariant model in Theorem 2.1.

Put

$$
\begin{gathered}
\psi_{1}(a, b, c)=a b+a c+b c \quad \psi_{2}(a, b, c)=a+b+c \\
g(a, b, c)=3 a^{2} b+3 a b^{2}+3 a^{2} c+3 a c^{2}+3 b^{2} c+3 b c^{2}+7 a b c
\end{gathered}
$$

and let us define the function $G: \mathbb{R}^{3} \longrightarrow \mathbb{R}^{3}$ as

$$
G(x, y, z)=\left(G_{1}(x, y, z), G_{2}(x, y, z), G_{3}(x, y, z)\right)
$$

with

$$
\begin{gathered}
G_{1}(x, y, z)=x^{2}+2 y z+x y+x z \quad G_{2}(x, y, z)=y^{2}+2 x z+x y+y z \\
G_{3}(x, y, z)=z^{2}+2 x y+x z+y z .
\end{gathered}
$$

Finally, for each $k \geq 3$, put $\psi_{k}(a, b, c)=\psi_{k-1}\left(G_{1}(a, b, c), G_{2}(a, b, c), G_{3}(a, b, c)\right)$, so that

$$
\psi_{k}(a, b, c)=\psi_{2}\left(G^{(k-2)}(a, b, c)\right)
$$

Theorem 2.12. The weighted generating functions $T_{n}(a, b, c), U_{n}(a, b, c), R_{n}(a, b, c)$, $L_{n}(a, b, c)$ and $Q_{n}(a, b, c)$ satisfying Equations (9), (10), (11), (12) and (13), with the initial conditions given in Theorem 2.10, are:

$$
\begin{gathered}
T_{n}(a, b, c)=2^{\frac{3^{n-1}-1}{2}} \prod_{k=1}^{n} \psi_{k}^{\frac{3^{n-k}+1}{2}}(a, b, c) \quad \text { for each } n \geq 1 ; \\
U_{n}(a, b, c)=2^{\frac{3^{n-1}-1}{2}} \prod_{k=1}^{n-1} \psi_{k}^{\frac{3^{n-k}-1}{2}}(a, b, c) G_{2}^{(n-1)}(a, b, c) \quad \text { for each } n \geq 2 ; \\
R_{n}(a, b, c)=2^{\frac{3^{n-1}-1}{2}} \prod_{k=1}^{n-1} \psi_{k}^{\frac{3^{n-k}-1}{2}}(a, b, c) G_{1}^{(n-1)}(a, b, c) \quad \text { for each } n \geq 2 ;
\end{gathered}
$$




$$
\begin{gathered}
L_{n}(a, b, c)=2^{\frac{3^{n-1}-1}{2}} \prod_{k=1}^{n-1} \psi_{k}^{\frac{3^{n-k}-1}{2}}(a, b, c) G_{3}^{(n-1)}(a, b, c) \quad \text { for each } n \geq 2 ; \\
Q_{n}(a, b, c)=2^{\frac{3^{n-1}-1}{2}} \prod_{k=1}^{n-2} \psi_{k}^{\frac{3^{n-k}-3}{2}}(a, b, c) g\left(G^{(n-2)}(a, b, c)\right) \quad \text { for each } n \geq 3,
\end{gathered}
$$

with $U_{1}(a, b, c)=b, R_{1}(a, b, c)=a, L_{1}(a, b, c)=c, Q_{1}(a, b, c)=1$ and $Q_{2}(a, b, c)=$ $2 g(a, b, c)$.

Proof. The proof is by induction on $n$. One can directly find:

$$
\begin{gathered}
T_{1}(a, b, c)=\psi_{1}(a, b, c) \quad U_{2}(a, b, c)=2 \psi_{1}(a, b, c) G_{2}(a, b, c) \\
R_{2}(a, b, c)=2 \psi_{1}(a, b, c) G_{1}(a, b, c) \quad L_{2}(a, b, c)=2 \psi_{1}(a, b, c) G_{3}(a, b, c) \\
Q_{3}(a, b, c)=2^{4} \psi_{1}^{3}(a, b, c) g\left(G_{1}(a, b, c), G_{2}(a, b, c), G_{3}(a, b, c)\right),
\end{gathered}
$$

and so the basis on the induction holds. We only prove the assertion for $T_{n}(a, b, c)$, by showing that Equation (9) is satisfied (the computations in the other cases are similar but more complicated). One has:

$$
\begin{aligned}
2 T_{n}^{2}\left(U_{n}+R_{n}+L_{n}\right)= & 2 \cdot 2^{3^{n-1}-1} \prod_{k=1}^{n} \psi_{k}^{3^{n-k}+1}(a, b, c) \cdot 2^{\frac{3^{n-1}-1}{2}} \prod_{k=1}^{n-1} \psi_{k}^{\frac{3^{n-k}-1}{2}}(a, b, c) \\
\cdot & \left(G_{2}^{(n-1)}(a, b, c)+G_{1}^{(n-1)}(a, b, c)+G_{3}^{(n-1)}(a, b, c)\right) \\
= & 2^{\frac{3^{n}-1}{2}} \prod_{k=1}^{n} \psi_{k}^{\frac{3^{n-k+1}+1}{2}}(a, b, c) \psi_{2}\left(G^{(n-1)}(a, b, c)\right) \\
= & 2^{\frac{3^{n}-1}{2}} \prod_{k=1}^{n+1} \psi_{k}^{\frac{3^{n-k+1}+1}{2}}(a, b, c)=T_{n+1} .
\end{aligned}
$$

Remark 2.13. Note that the function $g(a, b, c)$ coincides with the function $f(a, b, c)$, introduced in Section 2.2. However, the functions $G(x, y, z)$ and $F(x, y, z)$ do not coincide, which implies that the functions $\psi_{i}(a, b, c)$ and $\phi_{i}(a, b, c)$, factorizing the weighted generating functions of the spanning trees in the directional model and in the Schreier model, are different.

\section{Spanning trees on the Schreier graphs of the Hanoi Towers group}

In this section, we study spanning trees on the Schreier graphs of the Hanoi Towers group $H^{(3)}$, which are very similar to the Sierpiński graphs studied in the previous sections. We start with a combinatorial recursive approach as in Section 2, but this leads us to some 
equations that we can explicitly solve only in the unweighted case. Hence, in order to compute the weighted generating function, we follow a different approach: we use the self-similar presentation of the generators of the group to write the adjacency matrix of the graphs. Therefore, we are able to write the associated Laplace matrix and then we get the weighted generating function of the spanning trees by using a weighted version of Kirchhoff's Matrix-Tree Theorem. The self-similarity of the group is the key property that we use.

\subsection{The Schreier graphs of the Hanoi Towers group}

Recall that the Hanoi Towers group $H^{(3)}$ is generated by the automorphisms of the ternary rooted tree having the following self-similar form:

$$
a=(01)(i d, i d, a) \quad b=(02)(i d, b, i d) \quad c=(12)(c, i d, i d),
$$

where (01), (02) and (12) are elements of the symmetric group $\operatorname{Sym}(3)$, acting on the set $X=\{0,1,2\}$. Observe that $a, b, c$ are involutions. The associated Schreier graphs are self-similar in the sense of [16], that is, $\Sigma_{n+1}$ contains three copies of $\Sigma_{n}$ glued together by three edges, that we call "exceptional". By Definition 1.1, each vertex of $\Sigma_{n}$ corresponds to a word of length $n$ in the alphabet $X$. The graphs $\left\{\Sigma_{n}\right\}_{n \geq 1}$ can be recursively constructed via the following substitutional rules [11].

Rule I
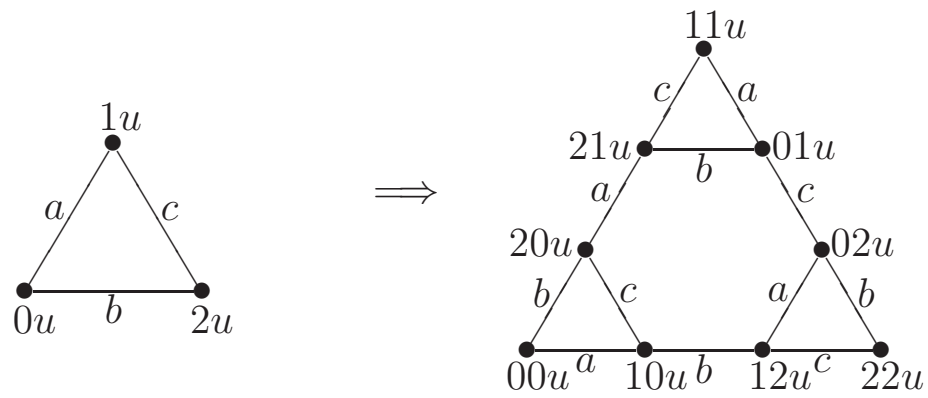

Rule II
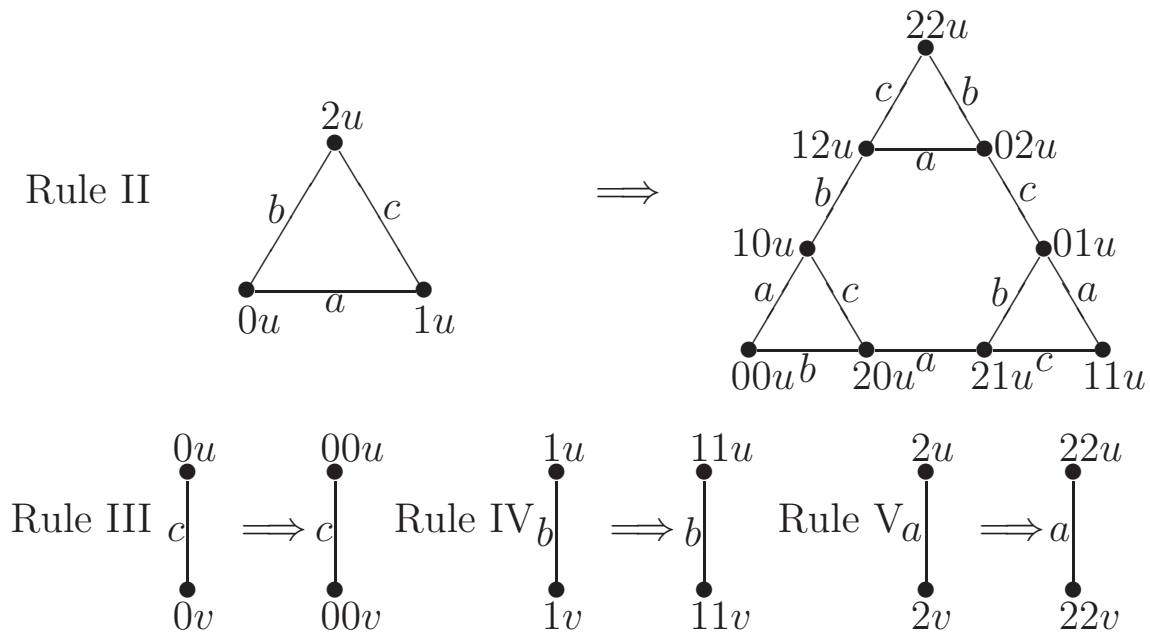
Notice that the word $u$ in Rules I and II can also be empty and the words $u$ and $v$ in Rules III, IV, $\mathrm{V}$ can also satisfy $u=v$ (in this case we get the three loops of $\Sigma_{n}$ ). The starting point of this recursive construction is the Schreier graph $\Sigma_{1}$ of the first level. We also draw a picture of $\Sigma_{2}$.
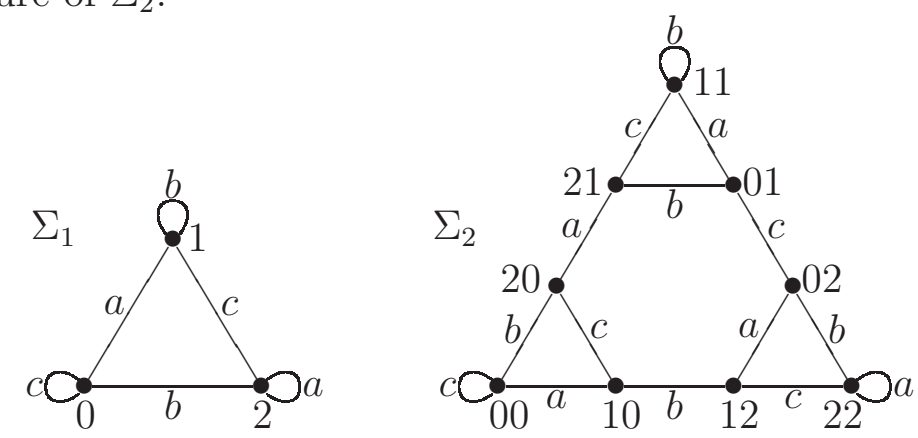

For each $n \geq 1$, the graph $\Sigma_{n+1}$ can be also obtained in the following recursive way: we take the union of three copies of $\Sigma_{n}$ and, for each one of the outmost vertices of $\Sigma_{n+1}$, the corresponding copy is reflected with respect to the bisectrix of the corresponding angle.

Remark 3.1. Observe that, for each $n \geq 1$, the graph $\Sigma_{n}$ has three loops, centered at the vertices $0^{n}, 1^{n}$ and $2^{n}$, labelled by $c, b$ and $a$, respectively. This is an easy consequence of the definition of the generators $a, b$ and $c$ of $H^{(3)}$. Moreover, these are the only loops in $\Sigma_{n}$. However, in what follows, we will be studying spanning trees on the Schreier graphs $\Sigma_{n}$ without loops, since a spanning tree of $\Sigma_{n}$ cannot contain any loop. By abuse of notation, we still denote by $\Sigma_{n}$ the graph without loops.

\subsection{Computation of the complexity}

For each $n \geq 1$, let $T_{n}(a, b, c), U_{n}(a, b, c), R_{n}(a, b, c), L_{n}(a, b, c)$ and $Q_{n}(a, b, c)$ be the generating functions having the same meaning as in Section 2. In what follows, we will often omit the argument $(a, b, c)$ in the generating functions.

Theorem 3.2. For each $n \geq 1$, the weighted generating functions $T_{n}(a, b, c), U_{n}(a, b, c)$, $R_{n}(a, b, c), L_{n}(a, b, c)$ and $Q_{n}(a, b, c)$ satisfy the following equations:

$$
\begin{aligned}
T_{n+1} & =T_{n}^{3}(a b+a c+b c)+2 a b c T_{n}^{2}\left(U_{n}+R_{n}+L_{n}\right) \\
U_{n+1} & =b T_{n}^{3}+T_{n}^{2}\left((a b+a c+b c) U_{n}+2 b\left(a R_{n}+c L_{n}\right)\right) \\
& +a b c T_{n}\left(3 R_{n} L_{n}+U_{n}\left(L_{n}+R_{n}+2 U_{n}\right)\right)+a b c T_{n}^{2} Q_{n} \\
R_{n+1} & =a T_{n}^{3}+T_{n}^{2}\left((a b+a c+b c) R_{n}+2 a\left(b U_{n}+c L_{n}\right)\right) \\
& +a b c T_{n}\left(3 U_{n} L_{n}+R_{n}\left(L_{n}+U_{n}+2 R_{n}\right)\right)+a b c T_{n}^{2} Q_{n} \\
& =c T_{n}^{3}+T_{n}^{2}\left((a b+a c+b c) L_{n}+2 c\left(a R_{n}+b U_{n}\right)\right) \\
L_{n+1} & +a b c T_{n}\left(3 R_{n} U_{n}+L_{n}\left(U_{n}+R_{n}+2 L_{n}\right)\right)+a b c T_{n}^{2} Q_{n}
\end{aligned}
$$




$$
\begin{aligned}
Q_{n+1} & =4 a b c T_{n} Q_{n}\left(U_{n}+R_{n}+L_{n}\right) \\
& +T_{n}^{2}\left((2 b+a+c) U_{n}+(2 a+b+c) R_{n}+(2 c+a+b) L_{n}\right) \\
& +T_{n}^{2} Q_{n}(a b+a c+b c)+T_{n}^{3} \\
& +2 a b c\left(U_{n}^{2}\left(R_{n}+L_{n}\right)+R_{n}^{2}\left(U_{n}+L_{n}\right)+L_{n}^{2}\left(U_{n}+R_{n}\right)+U_{n} R_{n} L_{n}\right) \\
& +2 T_{n}\left(U_{n} R_{n}(a c+b c+2 a b)+U_{n} L_{n}(a b+a c+2 b c)+R_{n} L_{n}(a b+b c+2 a c)\right. \\
& \left.+b U_{n}^{2}(a+c)+a R_{n}^{2}(b+c)+c L_{n}^{2}(a+b)\right),
\end{aligned}
$$

with initial conditions

$T_{1}(a, b, c)=a b+a c+b c \quad U_{1}(a, b, c)=b \quad R_{1}(a, b, c)=a \quad L_{1}(a, b, c)=c \quad Q_{1}(a, b, c)=1$.

Proof. The graph $\Sigma_{n+1}$ can be represented as

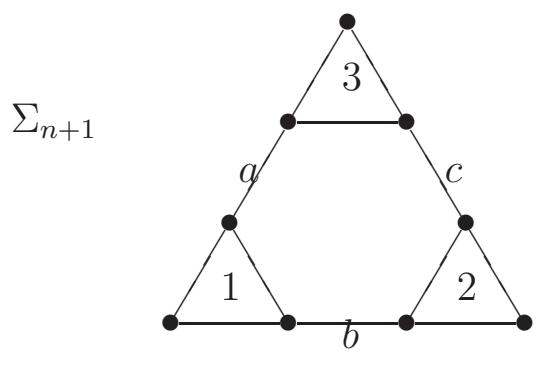

where the triangles 1, 2 and 3 represent subgraphs of $\Sigma_{n+1}$ isomorphic to the graph $\Sigma_{n}$. The edges labelled by $a, b$ and $c$ in the picture above are the exceptional edges joining the different copies of $\Sigma_{n}$. In the pictures of this proof we will use the same conventions as in Theorem 2.1.

Now, a spanning tree of $\Sigma_{n+1}$ can be obtained by choosing a spanning tree for each one of the triangles 1,2 and 3 , and omitting one of the edges $a, b$ and $c$ in order to have no cycle. This gives the contribution $T_{n}^{3}(a b+a c+b c)$ to $T_{n+1}$. A spanning tree of $\Sigma_{n+1}$ can also be obtained by choosing a spanning tree for two of the triangles 1, 2 and 3 , and a 2 -forest in the third triangle. This time, we do not need to omit any one of edges $a, b$ and $c$ (see the picture below).

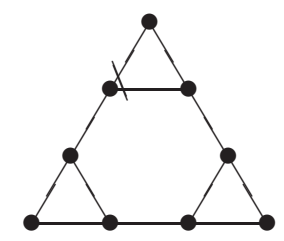

This situation corresponds to the contribution $2 a b c T_{n}^{2}\left(U_{n}+R_{n}+L_{n}\right)$ to $T_{n+1}$ and Equation (14) is proven.

We want to prove Equation (15). The two following pictures show that a spanning 2-forest of type $U_{n+1}$ can be obtained starting from three spanning trees of level $n$ (in this case we omit two exceptional edges), but also by taking two spanning trees in two copies of $\Sigma_{n}$ and a spanning 2-forest of type $U_{n}$ in the third one (by omitting one of the three exceptional edges). 

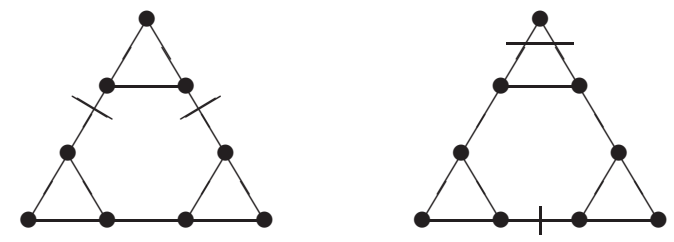

More precisely, the first picture corresponds to a contribution equal to $b T_{n}^{3}$, the second one to the contribution $T_{n}^{2} U_{n}(a b+a c+b c)$ to $U_{n+1}$. Consider now the following pictures.
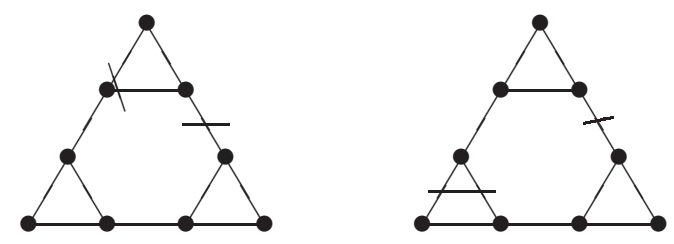

These configurations, together with their symmetric ones obtained by reflecting with respect to the vertical axis, give a contribution to $U_{n+1}$ equal to $2 b T_{n}^{2}\left(a R_{n}+c L_{n}\right)$. Consider now the two following pictures.
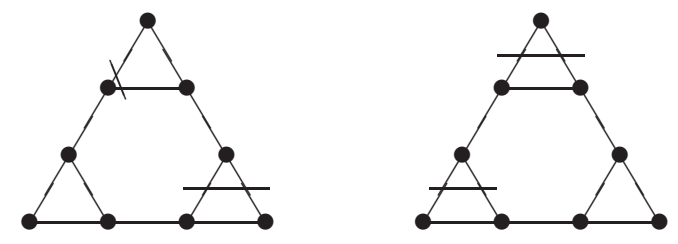

The left picture, together with its symmetric, gives the contribution $2 a b c T_{n} R_{n} L_{n}$; the right one, together with its symmetric, gives the contribution $a b c T_{n} U_{n}\left(R_{n}+L_{n}\right)$. Consider now the two following situations.
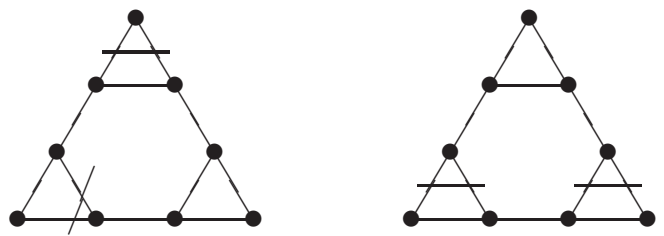

The picture on the left, together with its symmetric, contributes by $2 a b c T_{n} U_{n}^{2}$ to $U_{n+1}$. The picture on the right contributes by the summand $a b c T_{n} R_{n} L_{n}$. Finally, the contribution $a b c T_{n}^{2} Q_{n}$ is described by the following picture.

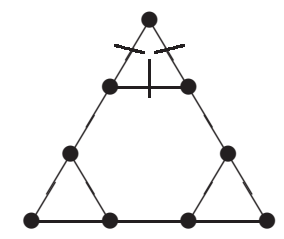

So we have proven Equation (15) about $U_{n+1}$. Equations (16) and (17) can be proven in a similar way.

We want to prove now Equation (18) about $Q_{n+1}$. Consider the following pictures. 

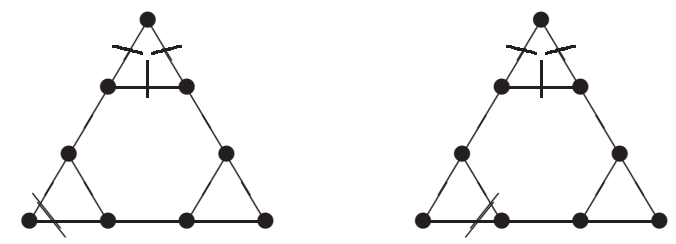

They provide, by symmetry, a contribution equal to $4 a b c T_{n} Q_{n}\left(U_{n}+R_{n}+L_{n}\right)$. Next, consider the following cases.
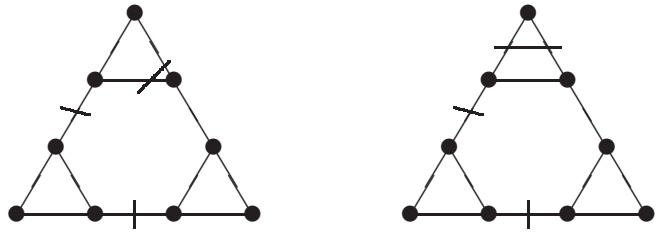

The picture on the left gives, by symmetry, a contribution equal to $2 T_{n}^{2}\left(b U_{n}+a R_{n}+c L_{n}\right)$. The picture on the right gives $T_{n}^{2}\left((a+c) U_{n}+(b+c) R_{n}+(a+b) L_{n}\right)$. Now consider the following cases.
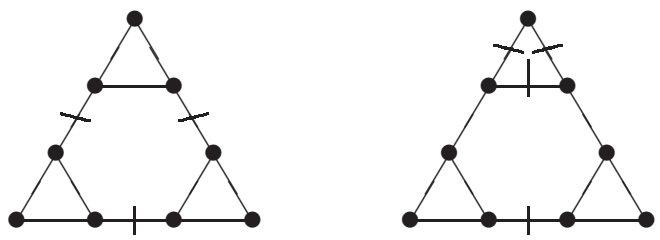

The picture on the left gives the contribution $T_{n}^{3}$ to $Q_{n+1}$, the picture on the right contributes by $T_{n}^{2} Q_{n}(a b+a c+b c)$. Consider the following pictures.
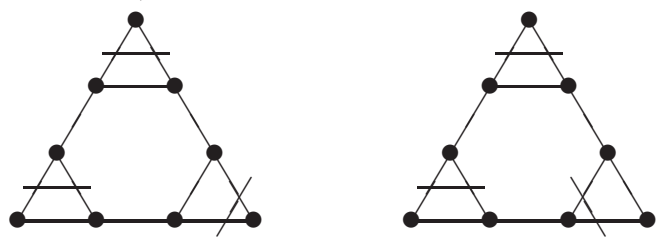

By symmetry, each one of the pictures above gives to $Q_{n+1}$ a contribution of

$$
a b c\left(U_{n}^{2}\left(R_{n}+L_{n}\right)+R_{n}^{2}\left(U_{n}+L_{n}\right)+L_{n}^{2}\left(U_{n}+R_{n}\right)\right) .
$$

Consider now the following pictures.
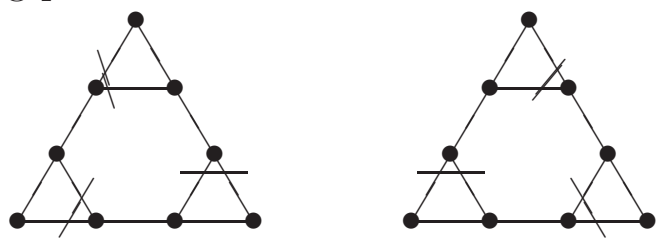

They give a contribution equal to $2 a b c U_{n} R_{n} L_{n}$ to $Q_{n+1}$. Now look at the following configurations. 

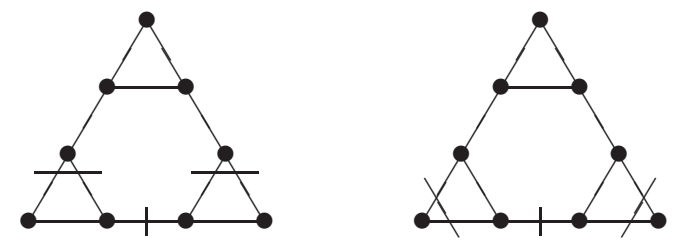

By symmetry, they correspond to the contribution $2 T_{n}\left(a b U_{n} R_{n}+b c U_{n} L_{n}+a c R_{n} L_{n}\right)$. The following picture gives the contribution $T_{n}\left(b U_{n}^{2}(a+c)+a R_{n}^{2}(b+c)+c L_{n}^{2}(a+b)\right)$.

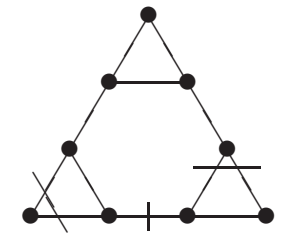

Finally, we have to consider the following four situations.
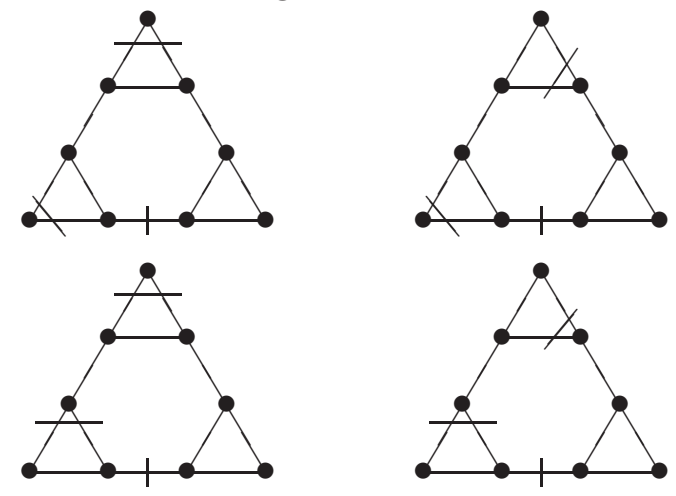

The first and the third pictures provide each a contribution equal to $T_{n}\left(U_{n} R_{n}(a+b) c+\right.$ $\left.U_{n} L_{n}(b+c) a+R_{n} L_{n}(a+c) b\right)$. The second one gives $T_{n}\left(b U_{n}^{2}(a+c)+a R_{n}^{2}(b+c)+c L_{n}^{2}(a+b)\right)$. The fourth one gives $2 T_{n}\left(a b U_{n} R_{n}+a c R_{n} L_{n}+b c U_{n} L_{n}\right)$. This completes the proof.

Equations (14), (15), (16), (17) and (18), with the initial conditions given in Theorem 3.2 , seem to be very hard to be explicitly solved. For this reason, in the next section we use a different strategy to find the weighted generating function. On the other hand, if we are interested in the complexity of these graphs, we can evaluate each generating function in $a=b=c=1$ and we get simpler equations. Let us define:

- $\tau_{n}:=\tau\left(\Sigma_{n}\right)=T_{n}(1,1,1)=$ complexity of $\Sigma_{n}$;

- $s_{n}:=U_{n}(1,1,1)=R_{n}(1,1,1)=L_{n}(1,1,1)=$ number of spanning 2-forests, where two fixed outmost vertices are in the same connected component and the third one lies in a different component;

- $q_{n}:=Q_{n}(1,1,1)=$ number of spanning 3 -forests, where each component contains exactly one outmost vertex. 
Corollary 3.3. For each $n \geq 1$, the values $\tau_{n}, s_{n}$ and $q_{n}$ satisfy the following relations:

$$
\begin{gathered}
\tau_{n+1}=3 \tau_{n}^{3}+6 \tau_{n}^{2} s_{n} \\
s_{n+1}=\tau_{n}^{3}+7 \tau_{n}^{2} s_{n}+7 \tau_{n} s_{n}^{2}+\tau_{n}^{2} q_{n} \\
q_{n+1}=3 \tau_{n}^{2} q_{n}+12 \tau_{n} s_{n} q_{n}+14 s_{n}^{3} \\
+12 \tau_{n}^{2} s_{n}+\tau_{n}^{3}+36 \tau_{n} s_{n}^{2},
\end{gathered}
$$

with initial conditions

$$
\tau_{1}=3 \quad s_{1}=q_{1}=1 .
$$

Proof. The proof follows from Theorem 3.2, by evaluating each function for $a=b=c=1$ and recalling that $U_{n}(1,1,1)=R_{n}(1,1,1)=L_{n}(1,1,1)$, for each $n \geq 1$.

Proposition 3.4. For every $n \geq 1$, the values $\tau_{n}, s_{n}$ and $q_{n}$ satisfying the relations given in Corollary 3.3 are:

$$
\begin{aligned}
& \text { 1. } \tau_{n}=3^{\frac{3^{n}+2 n-1}{4}} \cdot 5^{\frac{3^{n}-2 n-1}{4}} ; \\
& \text { 2. } s_{n}=3^{\frac{3^{n}-2 n-1}{4}} \cdot 5^{\frac{3^{n}-2 n-1}{4}} \cdot \frac{5^{n}-3^{n}}{2} ; \\
& \text { 3. } q_{n}=3^{\frac{3^{n}-6 n+3}{4}} \cdot 5^{\frac{3^{n}-2 n-1}{4}} \cdot\left(\frac{5^{n}-3^{n}}{2}\right)^{2}
\end{aligned}
$$

In particular, the asymptotic growth constant of the spanning trees of $\Sigma_{n}$ is $\frac{1}{4}(\log 3+\log 5)$.

Proof. The proof can be given by induction on $n$. Then, the asymptotic growth constant is obtained as the limit

$$
\lim _{n \rightarrow \infty} \frac{\log \left(\tau_{n}\right)}{\left|V\left(\Sigma_{n}\right)\right|}
$$

where $\left|V\left(\Sigma_{n}\right)\right|=3^{n}$ is the number of vertices of $\Sigma_{n}$, for each $n \geq 1$.

\subsection{Computation of the weighted generating function}

In this section, we compute the weighted generating function of the spanning trees on the graph $\Sigma_{n}$ by using the weighted version of the Kirchhoff's Theorem. The idea is to use the self-similar presentation of the generators of the group in order to describe recursively the adjacency matrix of the graph. The Laplace matrix of $\Sigma_{n}$ is then obtained as the difference between the degree matrix of $\Sigma_{n}$ and its adjacency matrix. By using the Schur complement Formula, one can compute a cofactor of the Laplace matrix passing from a square matrix of size $3^{n}$ to a square matrix of size $3^{n-1}$, which turns out to have the same structure of the original matrix, where the entries have been transformed via a rational function. This gives rise to a recursion process allowing to compute the cofactor. The same strategy is used in [8] to compute the partition function of the dimer model. See also [11], where the authors use the same idea to study the spectrum of the group $H^{(3)}$. 
Let $\Delta_{n}$ be the adjacency matrix of $\Sigma_{n}$, whose rows and columns are indexed by the vertices of $\Sigma_{n}$, i.e., words of length $n$ in the alphabet $\{0,1,2\}$, which are ordered lexicografically. Hence, $\Delta_{n}=a_{n}+b_{n}+c_{n}$, where the matrices $a_{n}, b_{n}$ and $c_{n}$ describe the action of the generators $a, b$ and $c$ of $H^{(3)}$, respectively, on the $n$-th level of the rooted ternary tree. In other words, we have:

$$
\left(a_{n}\right)_{i j}= \begin{cases}a & \text { if } a\left(v_{i}\right)=v_{j} \\ 0 & \text { otherwise }\end{cases}
$$

where $v_{i}$, for $i=1, \ldots, 3^{n}$, denotes the $i$-th vertex of $\Sigma_{n}$ with respect to the lexicografic order (similarly for $b_{n}$ and $c_{n}$ ). Notice that, in this way, we are writing the adjacency matrix of the graph with loops. However, in the Laplace matrix, the entries corresponding to loops will be deleted by using the degree matrix $(a+b+c) I_{n}$, according with the fact that a spanning tree cannot contain any loop.

The matrices $a_{n}, b_{n}$ and $c_{n}$ can be recursively represented, by using the self-similar description of the generators $a, b$ and $c$ of the group. For $n=1$, we set

$$
a_{1}=\left(\begin{array}{ccc}
0 & a & 0 \\
a & 0 & 0 \\
0 & 0 & a
\end{array}\right) \quad b_{1}=\left(\begin{array}{ccc}
0 & 0 & b \\
0 & b & 0 \\
b & 0 & 0
\end{array}\right) \quad c_{1}=\left(\begin{array}{ccc}
c & 0 & 0 \\
0 & 0 & c \\
0 & c & 0
\end{array}\right)
$$

and, for every $n>1$, we put

$$
a_{n}=\left(\begin{array}{ccc}
0 & a I_{n-1} & 0 \\
a I_{n-1} & 0 & 0 \\
0 & 0 & a_{n-1}
\end{array}\right) b_{n}=\left(\begin{array}{ccc}
0 & 0 & b I_{n-1} \\
0 & b_{n-1} & 0 \\
b I_{n-1} & 0 & 0
\end{array}\right) \quad c_{n}=\left(\begin{array}{ccc}
c_{n-1} & 0 & 0 \\
0 & 0 & c I_{n-1} \\
0 & c I_{n-1} & 0
\end{array}\right) .
$$

Therefore, Kirchhoff's Theorem states that the weighted generating function of the spanning trees on $\Sigma_{n}$ can be obtained by computing any cofactor of the Laplace matrix

$$
(a+b+c) I_{n}-\Delta_{n}=\left(\begin{array}{ccc}
(a+b+c) I_{n-1}-c_{n-1} & -a I_{n-1} & -b I_{n-1} \\
-a I_{n-1} & (a+b+c) I_{n-1}-b_{n-1} & -c I_{n-1} \\
-b I_{n-1} & -c I_{n-1} & (a+b+c) I_{n-1}-a_{n-1}
\end{array}\right) .
$$

We can choose, for instance, to compute the cofactor associated with the first row and the first column of the Laplace matrix. In order to compute it we put, for every $n>1$,

$$
\bar{\Delta}_{n}=\left(\begin{array}{ccc}
c_{n-1} & a I_{n-1}^{0} & b I_{n-1}^{0} \\
a I_{n-1}^{0} & b_{n-1} & c I_{n-1} \\
b I_{n-1}^{0} & c I_{n-1} & a_{n-1}
\end{array}\right)
$$

with

$$
I_{n}^{0}=I_{n}-\left(\begin{array}{cccc}
1 & 0 & \cdots & 0 \\
0 & 0 & \cdots & 0 \\
\vdots & 0 & \ddots & 0 \\
0 & 0 & 0 & 0
\end{array}\right)
$$


Moreover, we fix the notation $\gamma \widetilde{I}_{k}:=\gamma I_{k}^{0}+(a+b+c)\left(I_{k}-I_{k}^{0}\right)$, for each $k \geq 1$.

Define

$$
\Lambda_{n}:=(a+b+c) I_{n}-\bar{\Delta}_{n}=\left(\begin{array}{ccc}
(a+b+c) I_{n-1}-c_{n-1} & -a I_{n-1}^{0} & -b I_{n-1}^{0} \\
-a I_{n-1}^{0} & (a+b+c) I_{n-1}-b_{n-1} & -c I_{n-1} \\
-b I_{n-1}^{0} & -c I_{n-1} & (a+b+c) I_{n-1}-a_{n-1}
\end{array}\right) .
$$

The introduction of the matrices $I_{n}^{0}$ guarantees that $\frac{\operatorname{det}\left(\Lambda_{n}\right)}{a+b}$ is the generating function of the spanning trees of $\Sigma_{n}$, because we have performed all the necessary cancellations in $\bar{\Delta}_{n}$. More precisely, since $\left(\Lambda_{n}\right)_{11}=a+b$ and this is the only non-zero entry of the first row and column of $\Lambda_{n}$, it turns out that $\frac{\operatorname{det}\left(\Lambda_{n}\right)}{a+b}$ is equal to the cofactor of $(a+b+c) I_{n}-\Delta_{n}$ associated with the first row and the first column.

Moreover, we define the rational function $P: \mathbb{R}^{9} \longrightarrow \mathbb{R}^{9}$ as $P(\underline{x})=\left(P_{1}(\underline{x}), \ldots, P_{9}(\underline{x})\right)$, where

$$
P_{1}(\underline{x})=x_{1}, \quad P_{2}(\underline{x})=x_{2}, \quad P_{3}(\underline{x})=x_{3},
$$

$$
\begin{aligned}
P_{4}(\underline{x}) & =1 / D \\
& \cdot\left(x_{2} x_{3} x_{4}^{2} x_{5} x_{6} x_{9}+x_{1} x_{4} x_{5}^{2} x_{7} x_{8}^{2}-x_{1} x_{4}^{3} x_{5}^{2} x_{8}-x_{1} x_{2}^{2} x_{4} x_{5}^{2} x_{7}+x_{2} x_{3} x_{4}^{3} x_{9}^{2}+x_{1} x_{4}^{2} x_{5} x_{6} x_{7} x_{8}\right. \\
& -x_{1} x_{4}^{4} x_{5} x_{6}-x_{1}^{2} x_{2} x_{3} x_{4}^{3}+x_{1} x_{5} x_{6} x_{7}^{2} x_{8}^{2}-x_{1} x_{3}^{2} x_{5} x_{6} x_{8}^{2}-x_{1} x_{4}^{2} x_{5} x_{6} x_{7} x_{8}-x_{1} x_{2}^{2} x_{5} x_{6} x_{7}^{2} \\
& \left.+x_{1} x_{2}^{2} x_{3}^{2} x_{5} x_{6}+x_{2} x_{3} x_{4} x_{5}^{2} x_{6}^{2}-x_{1} x_{3}^{2} x_{4} x_{6}^{2} x_{8}+x_{1} x_{4} x_{6}^{2} x_{7}^{2} x_{8}-x_{1} x_{4}^{3} x_{6}^{2} x_{7}+x_{2} x_{3} x_{4}^{2} x_{5} x_{6} x_{9}\right)
\end{aligned}
$$

$P_{5}(\underline{x})=1 / D$

$$
\begin{aligned}
& \cdot\left(x_{1} x_{3} x_{5}^{3} x_{8}^{2}-x_{1} x_{2}^{2} x_{3} x_{5}^{3}-x_{2} x_{4} x_{5}^{4} x_{6}+x_{2} x_{4} x_{5}^{2} x_{6} x_{7} x_{9}+x_{2} x_{4}^{2} x_{5} x_{7} x_{9}^{2}-x_{2} x_{4} x_{5}^{2} x_{6} x_{7} x_{9}\right. \\
+ & x_{2} x_{4} x_{6} x_{7}^{2} x_{9}^{2}-x_{1}^{2} x_{2} x_{4}^{2} x_{5} x_{7}+x_{2} x_{5} x_{6}^{2} x_{7}^{2} x_{9}-x_{2} x_{3}^{2} x_{5} x_{6}^{2} x_{9}+x_{1} x_{3} x_{4} x_{5}^{2} x_{6} x_{8}-x_{2} x_{5}^{3} x_{6}^{2} x_{7} \\
+ & \left.x_{1} x_{3} x_{4} x_{5}^{2} x_{6} x_{8}-x_{2} x_{3}^{2} x_{4} x_{6} x_{9}^{2}-x_{1}^{2} x_{2} x_{4} x_{6} x_{7}^{2}+x_{1} x_{3} x_{4}^{2} x_{5} x_{6}^{2}+x_{1}^{2} x_{2} x_{3}^{2} x_{4} x_{6}-x_{2} x_{4}^{2} x_{5}^{3} x_{9}\right)
\end{aligned}
$$

$P_{6}(\underline{x})=1 / D$

- $\left(x_{3} x_{4} x_{5} x_{8}^{2} x_{9}^{2}-x_{2}^{2} x_{3} x_{4} x_{5} x_{9}^{2}-! x_{1}^{2} x_{3} x_{4} x_{5} x_{8}^{2}+x_{1}^{2} x_{2}^{2} x_{3} x_{4} x_{5}+x_{1} x_{2} x_{4}^{2} x_{5}^{2} x_{6}+x_{1} x_{2} x_{6}^{3} x_{7}^{2}\right.$

$+x_{3} x_{5}^{2} x_{6} x_{8}^{2} x_{9}-x_{3} x_{5}^{2} x_{6}^{3} x_{8}-x_{2}^{2} x_{3} x_{5}^{2} x_{6} x_{9}-x_{1}^{2} x_{3} x_{4}^{2} x_{6} x_{8}+x_{3} x_{4}^{2} x_{6} x_{8} x_{9}^{2}-x_{3} x_{4}^{2} x_{6}^{3} x_{9}$

$\left.-x_{3} x_{4} x_{5} x_{6}^{4}-x_{1} x_{2} x_{3}^{2} x_{6}^{3}+2 x_{1} x_{2} x_{4} x_{5} x_{6}^{2} x_{7}\right)$

$P_{7}(\underline{x})=x_{7}+1 / D$

- $\left(-x_{5} x_{7}^{2} x_{8}^{2} x_{9}+x_{3}^{2} x_{5} x_{7}^{2} x_{9}+x_{2}^{2} x_{5} x_{7}^{2} x_{9}-x_{2}^{2} x_{3}^{2} x_{5} x_{9}+x_{5}^{3} x_{7} x_{8}^{2}-x_{4}^{2} x_{5}^{3} x_{8}-x_{2}^{2} x_{5}^{3} x_{7}\right.$

$-x_{4} x_{7}^{2} x_{8} x_{9}^{2}+x_{4}^{2} x_{5} x_{7} x_{8} x_{9}+x_{3}^{2} x_{4} x_{8} x_{9}^{2}+x_{4}^{3} x_{7} x_{9}^{2}-x_{4}^{3} x_{5}^{2} x_{9}-x_{1}^{2} x_{3}^{2} x_{4} x_{8}+x_{1}^{2} x_{4} x_{7}^{2} x_{8}$

$+2 x_{3}^{2} x_{4} x_{5} x_{6} x_{8} x_{9}-2 x_{4} x_{5} x_{6} x_{7}^{2} x_{8} x_{9}+2 x_{4}^{3} x_{5} x_{6} x_{7} x_{9}+2 x_{4} x_{5}^{3} x_{6} x_{7} x_{8}-2 x_{4}^{3} x_{5}^{3} x_{6}$

$\left.-2 x_{1} x_{2} x_{3} x_{4}^{2} x_{5}^{2}+x_{4} x_{5}^{2} x_{7} x_{8} x_{9}-x_{1}^{2} x_{4}^{3} x_{7}\right)$

$P_{8}(\underline{x})=x_{8}+1 / D$

- $\left(-x_{4}^{2} x_{6}^{4} x_{7}-x_{3}^{2} x_{6}^{4} x_{8}-2 x_{4}^{3} x_{5} x_{6}^{3}-x_{4}^{4} x_{6}^{2} x_{9}-x_{1}^{2} x_{4}^{4} x_{8}+x_{4}^{4} x_{8} x_{9}^{2}+x_{2}^{2} x_{4}^{2} x_{7} x_{9}^{2}\right.$

$-x_{2}^{2} x_{3}^{2} x_{6}^{2} x_{9}+2 x_{4}^{2} x_{6}^{2} x_{7} x_{8} x_{9}-x_{1}^{2} x_{2}^{2} x_{4}^{2} x_{7}+x_{1}^{2} x_{4}^{2} x_{7} x_{8}^{2}-x_{4}^{2} x_{7} x_{8}^{2} x_{9}^{2}-2 x_{1} x_{2} x_{3} x_{4}^{2} x_{6}^{2}$

$-2 x_{4} x_{5} x_{6} x_{7} x_{8}^{2} x_{9}+2 x_{2}^{2} x_{4} x_{5} x_{6} x_{7} x_{9}-x_{6}^{2} x_{7}^{2} x_{8}^{2} x_{9}+x_{2}^{2} x_{6}^{2} x_{7}^{2} x_{9}+2 x_{4}^{3} x_{5} x_{6} x_{8} x_{9}$

$\left.+2 x_{4} x_{5} x_{6}^{3} x_{7} x_{8}+x_{6}^{4} x_{7}^{2} x_{8}+x_{3}^{2} x_{6}^{2} x_{8}^{2} x_{9}\right)$ 


$$
\begin{aligned}
P_{9}(\underline{x}) & =x_{9}+1 / D \\
& \cdot\left(x_{5}^{4} x_{8}^{2} x_{9}-x_{2}^{2} x_{5}^{4} x_{9}-x_{5}^{4} x_{6}^{2} x_{8}+x_{1}^{2} x_{5}^{2} x_{7} x_{8}^{2}-x_{1}^{2} x_{2}^{2} x_{5}^{2} x_{7}-x_{5}^{2} x_{7} x_{8}^{2} x_{9}^{2}+x_{2}^{2} x_{5}^{2} x_{7} x_{9}^{2}\right. \\
& -2 x_{1} x_{2} x_{3} x_{5}^{2} x_{6}^{2}+2 x_{4} x_{5}^{3} x_{6} x_{8} x_{9}-2 x_{4} x_{5}^{3} x_{6}^{3}+2 x_{1}^{2} x_{4} x_{5} x_{6} x_{7} x_{8}-2 x_{4} x_{5} x_{6} x_{7} x_{8} x_{9}^{2} \\
& -x_{3}^{2} x_{6}^{4} x_{9}-x_{5}^{2} x_{6}^{4} x_{7}+x_{1}^{2} x_{6}^{2} x_{7}^{2} x_{8}-x_{1}^{2} x_{3}^{2} x_{6}^{2} x_{8}-x_{6}^{2} x_{7}^{2} x_{8} x_{9}^{2}+x_{3}^{2} x_{6}^{2} x_{8} x_{9}^{2}+x_{6}^{4} x_{7}^{2} x_{9} \\
& \left.+2 x_{5}^{2} x_{6}^{2} x_{7} x_{8} x_{9}+2 x_{4} x_{5} x_{6}^{3} x_{7} x_{9}\right),
\end{aligned}
$$

with

$$
\begin{aligned}
D & =D\left(x_{1}, \ldots, x_{9}\right) \\
& =x_{7}^{2} x_{8}^{2} x_{9}^{2}-x_{3}^{2} x_{8}^{2} x_{9}^{2}-x_{4}^{2} x_{7} x_{8} x_{9}^{2}-x_{2}^{2} x_{7}^{2} x_{9}^{2}+x_{2}^{2} x_{3}^{2} x_{9}^{2}-x_{5}^{2} x_{7} x_{8}^{2} x_{9}+x_{3}^{2} x_{6}^{2} x_{8} x_{9} \\
& -x_{6}^{2} x_{7}^{2} x_{8} x_{9}+x_{4}^{2} x_{5}^{2} x_{8} x_{9}+x_{4}^{2} x_{6}^{2} x_{7} x_{9}+x_{2}^{2} x_{5}^{2} x_{7} x_{9}-x_{4}^{2} x_{7}^{2} x_{8}^{2}+x_{3}^{2} x_{4}^{2} x_{8}^{2} \\
& +x_{5}^{2} x_{6}^{2} x_{7} x_{8}+x_{4}^{4} x_{7} x_{8}-2 x_{1} x_{2} x_{3} x_{4} x_{5} x_{6}-x_{1}^{2} x_{2}^{2} x_{3}^{2}+x_{1}^{2} x_{2}^{2} x_{7}^{2}-x_{4}^{2} x_{5}^{2} x_{6}^{2} .
\end{aligned}
$$

Denote $P^{(k)}(\underline{x}):=P^{(k)}\left(x_{1}, \ldots, x_{9}\right)$ the $k$-th iteration of the function $P$ and use the notation

$$
P^{(k)}(a, b, c, a, b, c, a+b+c, a+b+c, a+b+c):=\left(a, b, c, a^{(k)}, b^{(k)}, c^{(k)}, e^{(k)}, f^{(k)}, g^{(k)}\right)
$$

and

$D^{(k)}:=D^{(k)}(a, b, c, a, b, c, a+b+c, a+b+c, a+b+c)=D\left(a, b, c, a^{(k)}, b^{(k)}, c^{(k)}, e^{(k)}, f^{(k)}, g^{(k)}\right)$,

where $a^{(0)}=a, b^{(0)}=b, c^{(0)}=c$ and $e^{(0)}=f^{(0)}=g^{(0)}=a+b+c$.

Moreover we define, for each $\underline{x} \in \mathbb{R}^{9}$,

$$
\Lambda_{k}(\underline{x})=\left(\begin{array}{ccc}
P_{7}(\underline{x}) \widetilde{I}_{k-1}-c_{k-1} & -P_{4}(\underline{x}) I_{k-1}^{0} & -P_{5}(\underline{x}) I_{k-1}^{0} \\
-P_{4}(\underline{x}) I_{k-1}^{0} & P_{8}(\underline{x}) I_{k-1}-b_{k-1} & -P_{6}(\underline{x}) I_{k-1} \\
-P_{5}(\underline{x}) I_{k-1}^{0} & -P_{6}(\underline{x}) I_{k-1} & P_{9}(\underline{x}) I_{k-1}-a_{k-1}
\end{array}\right) .
$$

Theorem 3.5. For each $n \geq 3$, the weighted generating function $T_{n}(a, b, c)$ of the spanning trees on the Schreier graph $\Sigma_{n}$ of the Hanoi Towers group $H^{(3)}$ is

$$
T_{n}(a, b, c)=\frac{1}{a+b} \prod_{k=0}^{n-3}\left(D^{(k)}\right)^{3^{n-k-2}} \cdot \operatorname{det}\left(\Lambda_{2}\left(P^{(n-3)}(a, b, c, a, b, c, a+b+c, a+b+c, a+b+c)\right)\right)
$$

Proof. It is clear, from what said above, that $T_{n}(a, b, c)=\frac{\operatorname{det}\left(\Lambda_{n}\right)}{a+b}$. More precisely, the factor $a+b$ corresponds to the entry $(1,1)$ of $\Lambda_{n}$. This entry must be simplified, since we want to compute the associated cofactor. If we expand twice the matrix $\Lambda_{n}$, using recursion, and we perform the transpositions (17) and (58) for both rows and columns, we get the matrix

$$
\left(\begin{array}{ll}
M_{11} & M_{12} \\
M_{21} & M_{22}
\end{array}\right)=
$$




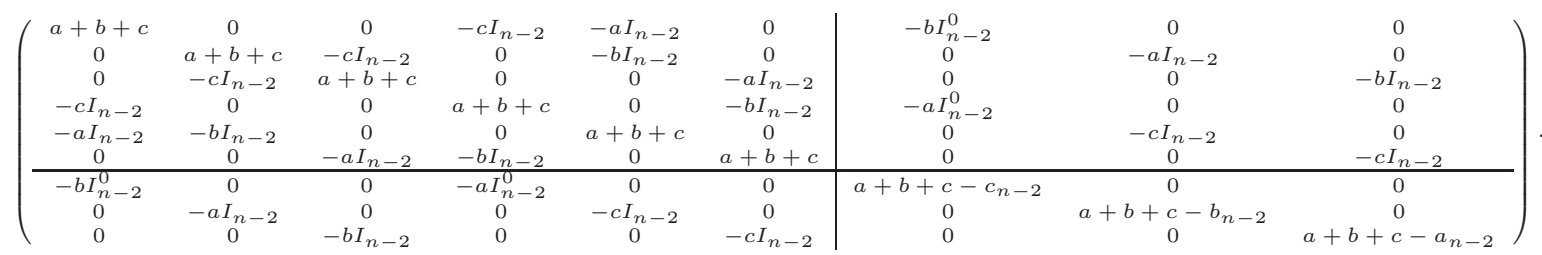

Note that each entry is a square matrix of size $3^{n-2}$ and $a+b+c$ is multiplied by $I_{n-2}$. Hence, the Schur complement Formula gives

$$
\begin{aligned}
\operatorname{det}\left(\Lambda_{n}\right) & =\operatorname{det}\left(M_{11}\right) \cdot \operatorname{det}\left(M_{22}-M_{21} M_{11}^{-1} M_{12}\right) \\
& =\left(D^{(0)}\right)^{3^{n-2}} \cdot \operatorname{det}\left(\begin{array}{ccc}
e^{(1)} \widetilde{I}_{n-2}-c_{n-2} & -a^{(1)} I_{n-2}^{0} & -b^{(1)} I_{n-2}^{0} \\
-a^{(1)} I_{n-2}^{0} & f^{(1)} I_{n-2}-b_{n-2} & -c^{(1)} I_{n-2} \\
-b^{(1)} I_{n-2}^{0} & -c^{(1)} I_{n-2} & g^{(1)} I_{n-2}-a_{n-2}
\end{array}\right) .
\end{aligned}
$$

The fundamental remark is that the matrix in Equation (19) has the same shape as $\Lambda_{n}$, since $e^{(0)} \widetilde{I}_{n-1}=(a+b+c) I_{n-1}$. Therefore, we can apply a recursive argument and use the same strategy $n-3$ times until we get a $9 \times 9$ matrix, that coincides with $\Lambda_{2}\left(P^{(n-3)}(a, b, c, a, b, c, a+b+c, a+b+c, a+b+c)\right)$.

Observe that the entry $(1,1)$ of $\Lambda_{2}\left(P^{(n-3)}(a, b, c, a, b, c, a+b+c, a+b+c, a+b+c)\right)$ is still equal to $a+b$; moreover, all the remaining entries in the first row and column of this matrix are zero. It follows that in the determinant of

$$
\Lambda_{2}\left(P^{(n-3)}(a, b, c, a, b, c, a+b+c, a+b+c, a+b+c)\right)
$$

a factor $(a+b)$ occurs, that we have to simplify in order to apply Kirchhoff's Theorem. Hence, Equation (19) becomes

$$
\begin{aligned}
\operatorname{det}\left(\Lambda_{n}\right) & =\left(D^{(0)}\right)^{3^{n-2}} \cdot \operatorname{det}\left(\Lambda_{n-1}(a, b, c, a, b, c, a+b+c, a+b+c, a+b+c)\right) \\
& =\left(D^{(0)}\right)^{3^{n-2}} \cdot\left(D^{(1)}\right)^{3^{n-3}} \cdot \operatorname{det}\left(\Lambda_{n-2}\left(P^{(1)}(a, b, c, a, b, c, a+b+c, a+b+c, a+b+c)\right)\right) \\
& =\prod_{k=0}^{n-3}\left(D^{(k)}\right)^{3^{n-k-2}} \cdot \operatorname{det}\left(\Lambda_{2}\left(P^{(n-3)}(a, b, c, a, b, c, a+b+c, a+b+c, a+b+c)\right)\right) \\
& =(a+b) T_{n}(a, b, c) .
\end{aligned}
$$

\section{Some statistics}

In this section, we deal with a statistical analysis about the number of edges, with a fixed label $w \in\{a, b, c\}$, occurring in a random spanning tree of the considered graph.

It is clear that in the case of the Schreier graphs $\left\{\Sigma_{n}\right\}_{n \geq 1}$ of the Hanoi Towers group, as in the case of both directional and Schreier labellings of the Sierpiński graphs $\left\{\Gamma_{n}\right\}_{n \geq 1}$, all the weights play the same role in the labelling of the edges of the graph.

On the other hand, in the rotational-invariant model, the weights $a$ and $b$ are symmetric, whereas the weight $c$ plays a special role in the labelling of the graph. Hence, it 
is interesting to perform such analysis on the Sierpiński graphs $\left\{\Gamma_{n}\right\}_{n \geq 1}$, when the edges are endowed with the rotational-invariant labelling.

Our techniques are classical: more precisely, logarithmic derivatives of the weighted generating function $T_{n}(a, b, c)$ with respect to $w$ give us the mean density of $w$-edges in a random spanning tree. We can further find the variance and show that the limiting distribution is normal.

Let $w_{n}$ be the random variable given by the number of edges labelled $w$ in a random spanning tree of $\Gamma_{n}$, with $w \in\{a, b, c\}$. Denote by $\mu_{n, w}$ and $\sigma_{n, w}^{2}$ the mean and the variance of $w_{n}$, respectively. From the remark above it follows that:

$$
\mu_{n, a}=\mu_{n, b} \quad \sigma_{n, a}^{2}=\sigma_{n, b}^{2} .
$$

Proposition 4.1. 1. The means and the variances of the random variables $a_{n}, b_{n}$ and $c_{n}$ are:

$$
\begin{aligned}
\mu_{n, a}=\mu_{n, b}=\frac{16 \cdot 3^{n}+7}{30} & \mu_{n, c}=\frac{13 \cdot 3^{n}+1}{30} \\
\sigma_{n, a}^{2}=\sigma_{n, b}^{2}=\frac{199 \cdot 3^{n}+28}{900} & \sigma_{n, c}^{2}=\frac{34 \cdot 3^{n}-2}{225} .
\end{aligned}
$$

2. The random variables $a_{n}, b_{n}$ and $c_{n}$ are asymptotically normal, as $n \rightarrow \infty$.

Proof. Let us prove the assertion for the random variable $c_{n}$ (similar computations can be done for $\left.a_{n}\right)$. Take the generating function $T_{n}(a, b, c)$ given in Theorem 2.2 and put:

$$
T_{n}(c):=T_{n}(1,1, c)=2^{\frac{3^{n}-1}{2}} 3^{\frac{3^{n}+2 n-1}{4}} 5^{\frac{3^{n-1}-2 n+1}{4}}(3 c+2)^{\frac{3^{n-1}-1}{2}}(2 c+1)^{\frac{3^{n}+1}{2}} .
$$

We can obtain the mean $\mu_{n, c}$ and the variance $\sigma_{n, c}^{2}$ of $c_{n}$ by studying the derivatives of the function $\log \left(T_{n}(c)\right)$. We get

$$
\mu_{n, c}=\left.\left(\log \left(T_{n}(c)\right)\right)^{\prime}\right|_{c=1}=\left.\frac{T_{n}^{\prime}(c)}{T_{n}(c)}\right|_{c=1}=\frac{13 \cdot 3^{n}+1}{30} .
$$

Taking once more derivative, one gets

$$
\left.\left(\log \left(T_{n}(c)\right)\right)^{\prime \prime}\right|_{c=1}=\left.\frac{T_{n}^{\prime \prime}(c) T_{n}(c)-\left(T_{n}^{\prime}(c)\right)^{2}}{\left(T_{n}(c)\right)^{2}}\right|_{c=1}=-\frac{127 \cdot 3^{n}+19}{450} .
$$

Hence,

$$
\sigma_{n, c}^{2}=\left.\left(\log \left(T_{n}(c)\right)\right)^{\prime \prime}\right|_{c=1}+\mu_{n, c}=\frac{34 \cdot 3^{n}-2}{225} .
$$

Next, let $C_{n}=\frac{c_{n}-\mu_{n, c}}{\sigma_{n, c}}$ be the normalized random variable; then the moment generating function of $C_{n}$ is given by

$$
\mathbb{E}\left(e^{t C_{n}}\right)=e^{-\mu_{n, c} t / \sigma_{n,}} \mathbb{E}\left(e^{t c_{n} / \sigma_{n, c}}\right)=e^{-\mu_{n, c} t / \sigma_{n, c}} \frac{T_{n}\left(e^{t / \sigma_{n, c}}\right)}{T_{n}(1)} .
$$


We get

$$
\mathbb{E}\left(e^{t C_{n}}\right)=3^{-\frac{3^{n}+1}{2}} 5^{-\frac{3^{n}-3}{6}} e^{-\frac{\left(13 \cdot 3^{n}+1\right) t}{2\left(34 \cdot 3^{n}-2\right)^{1 / 2}}}\left(2+3 e^{\frac{15 t}{\left(34 \cdot 3^{n}-2\right)^{1 / 2}}}\right)^{\frac{3^{n}-3}{6}}\left(1+2 e^{\frac{15 t}{\left(34 \cdot 3^{n}-2\right)^{1 / 2}}}\right)^{\frac{3^{n}+1}{2}}
$$

whose limit as $n \rightarrow \infty$ is $e^{\frac{t^{2}}{2}}$, showing that the random variable is asymptotically normal.

\section{Acknowledgements}

We wish to express our deepest gratitude to Tullio Ceccherini-Silberstein for useful comments and suggestions.

\section{References}

[1] R. Bacher, P. de la Harpe and T. Nagnibeda, The lattice of integral flows and the lattice of integral cuts on a finite graph, Bull. Soc. Math. France, 125, no. 2, (1997), 167-198.

[2] L. Bartholdi and R. Grigorchuk, On the spectrum of Hecke type operators related to some fractal groups, Tr. Mat. Inst. Steklova, 231, (2000), Din. Sist., Avtom. i Beskon. Gruppy, 5-45; translation in Proc. Steklov Inst. Math. 2000, no. 4 (231), $1-41$.

[3] L. Bartholdi, R. Grigorchuk and V. Nekrashevych, From fractal groups to fractal sets, in: "Fractals in Graz", 25-118, (P. Grabner and W. Woess editors), Trends in Mathematics, Birkäuser Verlag, Basel, 2003.

[4] N. Biggs, Chip-firing and the critical group of a graph, J. Algebraic Combin., 9, no. 1, (1999), 25-45.

[5] T. Ceccherini-Silberstein, A. Donno and D. Iacono, The Tutte Polynomial of the Schreier graphs of the Grigorchuk group and of the Basilica group, in: "Ischia Group Theory 2010", World Scientific Publishing, in press.

[6] S.-C. Chang, L.-C. Chen and W.-S. Yang, Spanning Trees on the Sierpinski Gasket, J. Stat. Phys., 126, No. 3, (2007), 649-667.

[7] D. D'Angeli, A. Donno and T. Nagnibeda, Partition functions of the Ising model on some self-similar Schreier graphs, to appear in the Proceedings of the Euroconference "Boundaries", Graz, June 2009, (W. Woess and F. Sobieczky editors), Birkhäuser, 2010; available at http://arxiv.org/abs/1003.0611

[8] D. D'Angeli, A. Donno and T. Nagnibeda, The dimer model on some self-similar Schreier graphs, in preparation.

[9] A. Donno and D. Iacono, The Tutte polynomial of some self-similar graphs, submitted; available at http://arxiv.org/abs/1006.5333 
[10] R. Grigorchuk, Solved and unsolved problems around one group, in: "Infinite groups: geometric, combinatorial and dynamical aspects", 117-218, (L. Bartholdi, T. Ceccherini-Silberstein, T. Smirnova-Nagnibeda and A. Żuk editors), Progr. Math., 248, Birkhäuser, Basel, 2005.

[11] R. Grigorchuk and Z. Sunić, Self-similarity and branching in group theory, in: "Groups St. Andrews 2005, I", London Math. Soc. Lecture Note Ser., 339, Cambridge Univ. Press, Cambridge, 2007, 36-95.

[12] D. Lorenzini, Smith normal form and Laplacians, J. Combin. Theory Ser. B, 98, no. 6, (2008), 1271-1300.

[13] R. Lyons, Asymptotic enumeration of spanning trees, Combin. Probab. Comput., 14, no. 4, (2005), 491-522.

[14] V. Nekrashevych, Self-similar Groups, Mathematical Surveys and Monographs, 117. American Mathematical Society, Providence, RI, 2005.

[15] E. Teufl and S. Wagner, The number of spanning trees of finite Sierpiński graphs, Proceedings of the Fourth Colloquium on Mathematics and Computer Science, Nancy, September 18-22, 2006, 411-414.

[16] E. Teufl and S. Wagner, Enumeration of matchings in families of self-similar graphs, to appear in Discrete Appl. Math.; available at http://www.math.uni-bielefeld.de/sfb701/preprints/sfb09014.pdf

[17] E. Teufl and S. Wagner, The number of spanning trees in self-similar graphs, submitted. 